\title{
Analysis of Trace Finite Element Methods for Surface Partial Differential Equations
}

\author{
Arnold Reusken*
}

Bericht Nr. 387

März 2014

Key words: trace finite elements, error analysis, elliptic surface equation

AMS Subject Classifications: 58J32, 65N15, 65N30

Institut für Geometrie und Praktische Mathematik RWTH Aachen

Templergraben 55, D-52056 Aachen (Germany)

* Institut für Geometrie und Praktische Mathematik, RWTH-Aachen University, D-52056 Aachen, Germany; e-mail: reusken@igpm.rwth-aachen.de 


\title{
ANALYSIS OF TRACE FINITE ELEMENT METHODS FOR SURFACE PARTIAL DIFFERENTIAL EQUATIONS
}

\author{
ARNOLD REUSKEN*
}

\begin{abstract}
In this paper we consider two variants of a trace finite element method for solving elliptic partial differential equations on a stationary smooth manifold $\Gamma$. A discretization error analysis for both methods in one general framework is presented. Higher order finite elements are treated and rather general numerical approximations $\Gamma_{h}$ of the manifold $\Gamma$ are allowed. Optimal order discretization error bounds are derived. Furthermore, the conditioning of the stiffness matrices is studied. It is proved that for one of these two variants the corresponding scaled stiffness matrix has a condition number $\sim h^{-2}$, independent of how $\Gamma_{h}$ intersects the outer triangulation.
\end{abstract}

Key words. trace finite elements, error analysis, elliptic surface equation

AMS subject classifications. 58J32, 65N15, 65N30

1. Introduction. Partial differential equations (PDEs) posed on evolving surfaces arise in many applications. In fluid dynamics, the concentration of surface active agents attached to an interface between two phases of immiscible fluids is governed by a transport-diffusion equation on the interface [17]. Another example is the diffusion of trans-membrane receptors in the membrane of a deforming and moving cell, which is typically modeled by a parabolic PDE posed on an evolving surface [2].

Recently, several numerical approaches for solving PDEs on surfaces have been introduced. The finite element method of Dziuk and Elliott [13] for the discretization of a PDE on an evolving surface is based on the Lagrangian description of a surface evolution and benefits from a special invariance property of test functions along material trajectories. If one considers the Eulerian description of a surface evolution, e.g., based on the level set method [26], then the surface is usually defined implicitly. In this case, regular surface triangulations and material trajectories of points on the surface are not easily available. Hence, Eulerian numerical techniques for the discretization of PDEs on surfaces have been studied in the literature. In $[1,27]$ numerical approaches were introduced that are based on extensions of PDEs off a two-dimensional surface to a three-dimensional neighbourhood of the surface. Then one can apply a standard finite element or finite difference disretization to treat the extended equation in $\mathbb{R}^{3}$. For a discussion of this extension approach we refer to $[16,14,6]$. A related approach was developed in [15], where advection-diffusion equations are numerically solved on evolving diffuse interfaces.

A different Eulerian technique for the numerical solution of an elliptic PDE posed on a stationary hypersurface in $\mathbb{R}^{3}$ was introduced in [24]. The main idea of this method is to use finite element spaces that are induced by the volume triangulations (tetrahedral decompositions) of a bulk domain in order to discretize a partial differential equation on the embedded surface. This method does not use an extension of the surface partial differential equation. It is instead based on a restriction (trace) of the outer finite element spaces to the (approximated) surface. This leads to discrete problems for which the number of degrees of freedom corresponds to the two-dimensional nature of the surface problem, similar to the Lagrangian approach. At the same time, the method is essentially Eulerian as the surface is not tracked by a surface mesh

*Institut für Geometrie und Praktische Mathematik, RWTH-Aachen University, D-52056 Aachen, Germany; email: reusken@igpm.rwth-aachen.de 
and may be defined implicitly as the zero level of a level set function. Optimal discretization error bounds were proved in [24]. The approach was further developed, for stationary surfaces, in $[10,22]$, where adaptive and streamline diffusion variants of this trace finite element method were introduced and analyzed. In the recent papers $[21,20]$ the trace method is extended to an Eulerian finite element method for the discretization of PDEs on evolving surfaces.

Recently, in [25, 7] related to this Eulerian trace finite element method the following interesting observation was made. If in this method with piecewise linears, the tangential gradients $\nabla_{\Gamma}$ used in the bilinear form are replaced by the full gradients $\nabla$, the method still has optimal convergence behavior. For the discretization of the Laplace-Beltrami equation on a stationary smooth surface $\Gamma$ we thus have the following two variants of the trace method: Find $u_{h}, u_{h}^{\Gamma} \in V_{h, m}^{\Gamma}$ such that

$$
\begin{aligned}
& \int_{\Gamma_{h}} \nabla u_{h} \cdot \nabla v_{h} \mathrm{~d} s_{h}=\int_{\Gamma_{h}} f_{h} v_{h} \mathrm{~d} s_{h} \quad \text { for all } v_{h} \in V_{h, m}^{\Gamma}, \\
& \int_{\Gamma_{h}} \nabla_{\Gamma_{h}} u_{h}^{\Gamma} \cdot \nabla_{\Gamma_{h}} v_{h} \mathrm{~d} s_{h}=\int_{\Gamma_{h}} f_{h} v_{h} \mathrm{~d} s_{h} \quad \text { for all } v_{h} \in V_{h, m}^{\Gamma} .
\end{aligned}
$$

with $V_{h, m}^{\Gamma}$ a trace finite element space (precise definition given below) with piecewise polynomials of degree $m, \Gamma_{h}$ an approximation of $\Gamma$, and $f_{h}$ an approximation of the exact data $f$. The method (1.2) is the original trace finite element method introduced and analyzed, for the case $m=1$, in [24]. The method (1.1) is introduced and analyzed, for the case $m=1$, in $[25,7]$. In the latter references it is shown that this method has optimal order of convergence for piecewise linear trace elements. The method (1.1) has two advantages compared to the one in (1.2). Firstly, it is more stable in the sense that $\left\|\nabla v_{h}\right\|_{L^{2}\left(\Gamma_{h}\right)} \geq\left\|\nabla_{\Gamma_{h}} v_{h}\right\|_{L^{2}\left(\Gamma_{h}\right)}$ holds. This affects the conditioning of the stiffness matrix, cf. discussion below. Secondly, if $\Gamma_{h}$ is given implicitly, the implementation of (1.1) is in general simpler than that of (1.1), because in the former we only have to evaluate functions on $\Gamma_{h}$ and we do not need any information about normals on $\Gamma_{h}$. On the other hand, although the two methods have the same order of convergence, the discretization error in $u_{h}$ is in general larger than in $u_{h}^{\Gamma}$.

The two main contributions of this paper are the following. Firstly, we present a discretization error analysis of both methods in one general framework. We do not restrict to the case $m=1$, but allow arbitrary degree $m$ finite element polynomials. Furthermore, we do not consider a specific construction of $\Gamma_{h}$ (e.g. by interpolating $\Gamma$ or by using level set functions) but only assume that $\Gamma_{h}$ satisfies certain accuracy conditions, e.g. $\operatorname{dist}\left(\Gamma_{h}, \Gamma\right) \leq c h^{k+1}$ and $\left\|n-n_{h}\right\|_{L^{\infty}\left(\Gamma_{h}\right)} \leq c h^{k}$ (with $n, n_{h}$ the normals on $\Gamma, \Gamma_{h}$ ). The analysis explains why in general the method (1.1) can be expected to be less accurate than (1.2). Furthermore, the analysis reveals the different roles of the data approximation error (replacing $f$ by $f_{h}$ ), the finite element approximation error (quality of $V_{h, m}$ ) and the geometric error (approximation of $\Gamma$ by $\Gamma_{h}$ ). We derive optimal error bounds both in $H^{1}$ and $L^{2}$-norms, e.g. $\left\|u^{e}-u_{h}^{(\Gamma)}\right\|_{L^{2}\left(\Gamma_{h}\right)} \leq$ $c\left(h^{m+1}+h^{k+1}\right)$. To our knowledge, neither for (1.1) nor for (1.2) error bounds for $m \geq 2$ are known in the literature. Related to the geometric error we assume that the integrals in (1.1) and (1.2) can be determined exactly. In practice, for the case of higher order approximations $\Gamma_{h}$ of $\Gamma$ (i.e., $k \geq 2$ ) this is often not a realistic assumption. If the exact distance function to $\Gamma$ is known, one can use polynomial approximations $\Gamma_{h}$ as presented in [8] to satisfy this assumption. If, however, $\Gamma$ is given implicitly (via a level set function) it is not clear how to satisfy this assumption. 
This topic related to quadrature errors in the evaluation of the integrals in (1.1) and (1.2) will be treated in a forthcoming paper.

The second main contribution is related to linear algebra aspects. For this, we restrict to the case $m=1$. For the trace finite element method, the conditioning properties of the mass and stiffness matrices are different from that of standard finite element discretizations of elliptic problems. This topic is addressed in [23]. Only if certain (fairly reasonable) conditions on how the approximate surface $\Gamma_{h}$ intersects the outer volume triangulation are fulfilled, the diagonally scaled mass matrix for $V_{h, 1}$ and the diagonally scaled stiffness matrix for (1.2) have condition numbers that behave like $h^{-2}$. Recently, in [5] a stabilization procedure for the discretization (1.2) has been introduced which results in a stiffness matrix with a condition number $\sim h^{-2}$, independent of how $\Gamma_{h}$ intersects the outer volume triangulation. As mentioned above, the discretization (1.1) is more stable than (1.2). In particular, the conditioning of the stiffness matrix corresponding to (1.1) is better than that of the one corresponding to (1.2). We prove that, without any stabilization, the stiffness matrix for (1.2), with an appropriate scaling, has a condition number $\sim h^{-2}$, independent of how $\Gamma_{h}$ intersects the outer volume triangulation. As far as we know, linear algebra aspects related to (1.2) have not been studied in the literature, yet.

We include a section with results of a numerical experiment in which, for the case $m=k=1$, the two methods are compared.

2. Laplace-Beltrami equation and finite element discretizations. As a model problem for an elliptic equation we consider the pure diffusion (i.e., LaplaceBeltrami) equation. We assume that $\Omega$ is an open subset in $\mathbb{R}^{3}$ which contains a connected compact smooth hypersurface $\Gamma$ without boundary. The (outward pointing) normal on $\Gamma$ is denoted by $n_{\Gamma}$. For a sufficiently smooth function $g: \Omega \rightarrow \mathbb{R}$ the tangential derivative is defined by

$$
\nabla_{\Gamma} g=\left(I-n_{\Gamma} n_{\Gamma}^{T}\right) \nabla g
$$

By $\Delta_{\Gamma}=\nabla_{\Gamma} \cdot \nabla_{\Gamma}$ we denote the Laplace-Beltrami operator on $\Gamma$. We consider the Laplace-Beltrami problem in weak form: For given $f \in L^{2}(\Gamma)$ with $\int_{\Gamma} f \mathrm{~d} s=0$, determine $u \in H^{1}(\Gamma)$ with $\int_{\Gamma} u \mathrm{~d} s=0$ such that

$$
\int_{\Gamma} \nabla_{\Gamma} u \cdot \nabla_{\Gamma} v \mathrm{~d} s=\int_{\Gamma} f v \mathrm{~d} s \quad \text { for all } v \in H^{1}(\Gamma) .
$$

The solution $u$ is unique and satisfies $u \in H^{2}(\Gamma)$ with $\|u\|_{H^{2}(\Gamma)} \leq c\|f\|_{L^{2}(\Gamma)}$ and a constant $c$ independent of $f$, cf. [12].

We introduce two trace finite element methods for the discretization of this equation. Let $\left\{\mathcal{T}_{h}\right\}_{h>0}$ be a family of tetrahedral triangulations of the domain $\Omega \subset \mathbb{R}^{3}$ that contains $\Gamma$. These triangulations are assumed to be regular, consistent and stable [3]. Given $\mathcal{T}_{h}$, we need an approximation $\Gamma_{h}$ of $\Gamma$. Possible constructions of $\Gamma_{h}$ and precise conditions that $\Gamma_{h}$ has to satisfy will be discussed further on. For the definition of the method, we (only) assume that $\Gamma_{h}$ is a Lipschitz hypersurface without boundary, which is "close to" $\Gamma$. The local triangulation $\mathcal{T}_{h}^{\Gamma} \subset \mathcal{T}_{h}$ is defined by $\mathcal{T}_{h}^{\Gamma}=\left\{T \in \mathcal{T}_{h} \mid\right.$ meas $\left._{2}\left(\Gamma_{h} \cap T\right)>0\right\}$. If $\Gamma_{h} \cap T$ consists of a face $F$ of $T$, we include in $\mathcal{T}_{h}^{\Gamma}$ only one of the two tetrahedra which have this $F$ as their intersection. The domain formed by the triangulation $\mathcal{T}_{h}^{\Gamma}$ is denoted by $\omega_{h}$. On the local domain $\omega_{h}$ we define the standard space of $H^{1}$-conforming finite elements, with finite elements of degree $m \geq 1$ :

$$
V_{h, m}:=\left\{v_{h} \in C\left(\omega_{h}\right) \mid v_{h \mid T} \in \mathcal{P}_{m} \text { for all } T \in \mathcal{T}_{h}^{\Gamma}\right\} .
$$


We also define the corresponding trace space:

$$
V_{h, m}^{\Gamma}:=\left\{v_{h \mid \Gamma_{h}} \mid v_{h} \in V_{h, m}\right\}, \quad V_{h, m}^{\Gamma, 0}:=\left\{v_{h} \in V_{h, m}^{\Gamma} \mid \int_{\Gamma_{h}} v_{h} \mathrm{~d} s_{h}=0\right\} .
$$

An elementary but important observation is that for $v_{h} \in V_{h, m}^{\Gamma}$ its gradient $\nabla\left(v_{h \mid \Gamma_{h}}\right)=$ $\nabla v_{h}$ is well-defined on $\Gamma_{h}$. On $\Gamma_{h}$ we need an approximation of the data $f$, denoted by $f_{h}$. We assume that $\int_{\Gamma_{h}} f_{h} \mathrm{~d} s_{h}=0$ holds. In this paper we consider the following two discretization methods: Find $u_{h} \in V_{h, m}^{\Gamma, 0}$ such that

$$
\int_{\Gamma_{h}} \nabla u_{h} \cdot \nabla v_{h} \mathrm{~d} s_{h}=\int_{\Gamma_{h}} f_{h} v_{h} \mathrm{~d} s_{h} \quad \text { for all } v_{h} \in V_{h, m}^{\Gamma},
$$

and: Find $u_{h}^{\Gamma} \in V_{h, m}^{\Gamma, 0}$ such that

$$
\int_{\Gamma_{h}} \nabla_{\Gamma_{h}} u_{h}^{\Gamma} \cdot \nabla_{\Gamma_{h}} v_{h} \mathrm{~d} s_{h}=\int_{\Gamma_{h}} f_{h} v_{h} \mathrm{~d} s_{h} \quad \text { for all } v_{h} \in V_{h, m}^{\Gamma}
$$

These discrete problems have unique solutions. This follows from $\left\|\nabla_{\Gamma_{h}} v_{h}\right\|_{L^{2}\left(\Gamma_{h}\right)} \leq$ $\left\|\nabla v_{h}\right\|_{L^{2}\left(\Gamma_{h}\right)}$ and the fact that $\left\|\nabla_{\Gamma_{h}} v_{h}\right\|_{L^{2}\left(\Gamma_{h}\right)}=0$ implies that $v_{h}$ is constant on $\Gamma_{h}$.

3. Preliminaries. In the analysis of the methods introduced above we always assume that $\Gamma$ is sufficiently smooth. We do not specify the required smoothness of $\Gamma$. The signed distance function to $\Gamma$ is denoted by $d$, with $d$ negative in the interior of $\Gamma$. On

$$
U_{\delta}:=\left\{\mathbf{x} \in \mathbb{R}^{3}|| d(x) \mid<\delta\right\},
$$

with $\delta>0$ sufficiently small, we define

$$
\begin{aligned}
& n(x)=\nabla d(x), H(x)=D^{2} d(x), \quad P(x)=I-n(x) n(x)^{T}, \\
& p(x)=x-d(x) n(x), \quad v^{e}(x)=v(p(x)) \text { for } v \text { defined on } \Gamma .
\end{aligned}
$$

The eigenvalues of $H(x)$ are denoted by $\kappa_{1}(x), \kappa_{2}(x)$ and 0 . Note that $v^{e}$ is simply the constant extension of $v$ (given on $\Gamma$ ) along the normals $n$. The tangential derivative can be written as $\nabla_{\Gamma} g(x)=P(x) \nabla g(x)$ for $x \in \Gamma$. We assume $\delta_{0}>0$ to be sufficiently small such that on $U_{\delta_{0}}$ the decomposition

$$
x=p(x)+d(x) n(x)
$$

is unique for all $x \in U_{\delta_{0}}$. In the remainder we only consider $U_{\delta}$ with $0<\delta \leq \delta_{0}$. In the analysis we use the following formulas from [9]:

$$
\begin{aligned}
\nabla u^{e}(x) & =(I-d(x) H(x)) \nabla_{\Gamma} u(p(x)) \quad \text { a.e on } U_{\delta_{0}}, u \in H^{1}(\Gamma), \\
\kappa_{i}(\mathbf{x}) & =\frac{\kappa_{i}(p(x))}{1+d(x) \kappa_{i}(p(x))}, \quad \text { for } x \in U_{\delta_{0}}, \quad i=1,2 .
\end{aligned}
$$

The first one follows from differentiating the relation $u^{e}(x)=u(p(x))$ and using $\nabla p(x)=P(x)-d(x) H(x)$. Using the result (3.5) one obtains that if $\delta \in\left(0, \delta_{0}\right]$ satisfies

$$
5 \delta<\left(\max _{i=1,2}\left\|\kappa_{i}\right\|_{L^{\infty}(\Gamma)}\right)^{-1}
$$


then

$$
\|d\|_{L^{\infty}\left(U_{\delta}\right)} \max _{i=1,2}\left\|\kappa_{i}\right\|_{L^{\infty}\left(U_{\delta}\right)} \leq \frac{1}{4}
$$

holds. In the following lemma Sobolev norms on $U_{\delta}$ of the normal extension $u^{e}$ are related to corresponding norms on $\Gamma$. Such results are known in the literature, e.g. $[12,9]$. For completeness we include a proof. Note that these results only involve $\Gamma$ and its neighborhood $U_{\delta}$. The approximate surface $\Gamma_{h}$ does not play a role.

Lemma 3.1. Let (3.6) be satisfied. For all $u \in H^{m}(\Gamma)$ the following holds:

$$
\left\|D^{\mu} u^{e}\right\|_{L^{2}\left(U_{\delta}\right)} \leq c \sqrt{\delta}\|u\|_{H^{m}(\Gamma)}, \quad|\mu|=m \geq 0,
$$

with a cosntant $c$ independent of $\delta$ and $u$.

Proof. Define

$$
\mu(x):=\left(1-d(x) \kappa_{1}(x)\right)\left(1-d(x) \kappa_{2}(x)\right), \quad x \in U_{\delta} .
$$

From (2.20), (2.23) in [9] we have

$$
\mu(x) \mathrm{d} x=\mathrm{d} r \mathrm{~d} s(p(x)), \quad x \in U_{\delta},
$$

where $\mathrm{d} x$ is the volume measure in $U_{\delta}, \mathrm{d} s$ the surface measure on $\Gamma$ and $r$ the local coordinate at $x \in \Gamma$ in the direction $n(p(x))=n(x)$. Using (3.7) we get

$$
\frac{9}{16} \leq \mu(x) \leq \frac{25}{16} \text { for all } x \in U_{\delta} .
$$

Using the local coordinate representation $x=(p(x), r)$, for $x \in U_{\delta}$, we have

$$
\begin{aligned}
\int_{U_{\delta}} u^{e}(x)^{2} \mu(x) \mathrm{d} x & =\int_{-\delta}^{\delta} \int_{\Gamma}\left[u^{e}(p(x), r)\right]^{2} \mathrm{~d} s(p(x)) \mathrm{d} r \\
& =\int_{-\delta}^{\delta} \int_{\Gamma}[u(p(x), 0)]^{2} \mathrm{~d} s(p(x)) \mathrm{d} r=2 \delta\|u\|_{L^{2}(\Gamma)}^{2} .
\end{aligned}
$$

Combining this with (3.9) yields the result for $m=0$. Using (3.4) we get

$$
\int_{U_{\delta}}\left[\nabla u^{e}(x)\right]^{2} \mu(x) \mathrm{d} x=\int_{-\delta}^{\delta} \int_{\Gamma}\left[(I-d(x) H(x)) \nabla_{\Gamma} u(p(x))\right]^{2} \mathrm{~d} s(p(x)) \mathrm{d} r .
$$

In combination with $\|I-d H\|_{L^{\infty}\left(U_{\delta}\right)} \leq c$ we obtain the result for $m=1$. For $m \geq 2$ the same argument can be applied repeatedly if we differentiate (3.4) and use the chain rule.

In the remainder we assume that $\delta_{0}$ is sufficiently small such that it satisfies (3.6). that

4. Approximation error bounds. From $\left\|\nabla_{\Gamma_{h}} v(x)\right\| \leq\|\nabla v(x)\|$, it follows

$$
\begin{aligned}
& \min _{v_{h} \in V_{h, m}^{\Gamma}}\left(\left\|u^{e}-v_{h}\right\|_{L^{2}\left(\Gamma_{h}\right)}+h\left\|\nabla_{\Gamma_{h}}\left(u^{e}-v_{h}\right)\right\|_{L^{2}\left(\Gamma_{h}\right)}\right) \\
& \leq \min _{v_{h} \in V_{h, m}^{\Gamma}}\left(\left\|u^{e}-v_{h}\right\|_{L^{2}\left(\Gamma_{h}\right)}+h\left\|\nabla\left(u^{e}-v_{h}\right)\right\|_{L^{2}\left(\Gamma_{h}\right)}\right)
\end{aligned}
$$

holds. In this section we derive bounds for the approximation error on the right-hand side. The analysis is simpler than the one presented in [24]. This is due to lemma 4.1 
below, which was not used in [24]. Furthermore, in [24] only $m=1$ (linear finite elements) is treated, whereas below we treat $m \geq 1$.

For the derivation of an optimal approximation error bound we need some mild assumptions on the family of approximate surfaces $\left\{\Gamma_{h}\right\}_{h>0}$, in particular on how $\Gamma_{h}$ is related to the triangulation $\mathcal{T}_{h}$. In Remark 1 we discuss a few standard cases in which these assumptions are satisfied. The closed connected Lipschitz manifold $\Gamma_{h}$ can be partitioned as follows:

$$
\Gamma_{h}=\cup_{T \in \mathcal{T}_{h}^{\Gamma}} \Gamma_{T}, \quad \Gamma_{T}:=\Gamma_{h} \cap T .
$$

The unit normal (pointing outward from the interior of $\Gamma_{h}$ ) is denoted by $n_{h}(x)$, and is defined a.e. on $\Gamma_{h}$.

Assumption 1. (A1) We assume that there is a constant $c_{0}$ independent of $h$ such for the local domain $\omega_{h}$ we have

$$
\omega_{h} \subset U_{\delta}, \quad \text { with } \delta=c_{0} h \leq \delta_{0} .
$$

(A2) We assume that for each $T \in \mathcal{T}_{h}^{\Gamma}$ the local surface section $\Gamma_{T}$ consists of connected parts $\Gamma_{T}^{(i)}, i=1, \ldots p$, such that $\partial \Gamma_{T}^{(i)} \cap \partial T$ is a simple closed curve and $\left\|n_{h}(x)-n_{h}(y)\right\| \leq c_{1} h$ holds for $x, y \in \partial \Gamma_{T}^{(i)}$. The number $p$ and constant $c_{1}$ are uniformly bounded w.r.t. $h$ and $T \in \mathcal{T}_{h}$.

REMARK 1 . The condition (A1) essentially means that $\operatorname{dist}\left(\Gamma_{h}, \Gamma\right) \leq c_{0} h$ holds, which is a very mild condition on the accuracy of $\Gamma_{h}$ as an approximation of $\Gamma$. The condition ensures that the local triangulation $\mathcal{T}_{h}^{\Gamma}$ has sufficient resolution for representing the surface $\Gamma$ approximately. The condition (A2) allows multiple intersections (namely $p$ ) of $\Gamma_{h}$ with one tetrahedron $T \in \mathcal{T}_{h}^{\Gamma}$. An illustration for the two-dimensional case is shown in Figure 4.1. We discuss three situations in which Assumption 1 is satisfied. For the case $\Gamma_{h}=\Gamma$ and with $h$ sufficiently small the conditions in Assumption 1 hold. If $\Gamma_{h}$ is a shape-regular triangulation, consisting of triangles with diameter $\mathcal{O}(h)$ and vertices on $\Gamma$, then for $h$ sufficiently small the conditions are satisfied. Finally, consider the case in which $\Gamma$ is the zero level of a smooth level set function $\phi$ and $\phi_{h}$ is a finite element approximation of $\phi$, on the triangulation $\mathcal{T}_{h}$. Let $\Gamma_{h}$ be the zero level of $\phi_{h}$. If $\left\|\phi-\phi_{h}\right\|_{L^{\infty}\left(\omega_{h}\right)}+h\left\|\nabla\left(\phi-\phi_{h}\right)\right\|_{L^{\infty}\left(\omega_{h}\right)} \leq c h^{2}$ holds, then the conditions are satisfied, provided $h$ is sufficiently small.
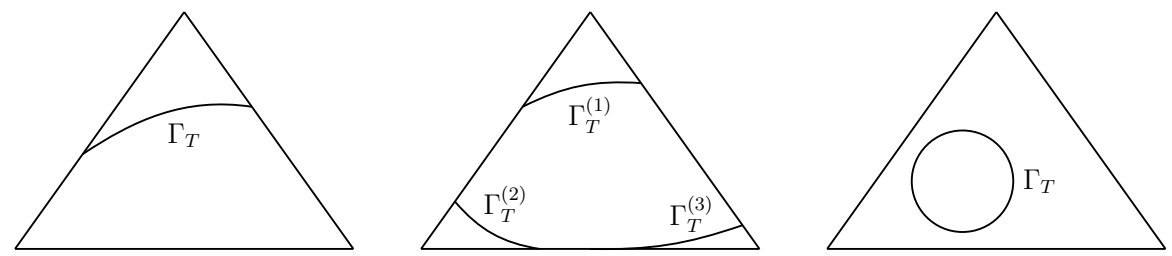

FIG. 4.1. Illustration of local surface sections $\Gamma_{T}^{(i)}$, cf. Assumption 1 (A2). The left picture is the generic case $(p=1)$; the middle picture has $p=3$ intersections; the situation in the right picture is not allowed.

A (slightly) simplified version of the following lemma in presented in $[18,19]$.

Lemma 4.1. Let (A2) in Assumption 1 be satisfied. There exists a constant $c$, independent of $h$ and of how $\Gamma_{h}$ intersects $\mathcal{T}_{h}^{\Gamma}$, such that for all $T \in \Gamma_{h}^{\Gamma}$ and all 
$v \in H^{1}(T)$ the following holds, with $h_{T}:=\operatorname{diam}(T)$ :

$$
\|v\|_{L^{2}\left(\Gamma_{T}\right)}^{2} \leq c\left(h_{T}^{-1}\|v\|_{L^{2}(T)}^{2}+h_{T}\|\nabla v\|_{L^{2}(T)}^{2}\right) .
$$

Proof. Since $\mathcal{T}_{h}^{\Gamma}$ is a shape regular triangulation, there is a constant independent of $h$ such that

$$
\|v\|_{L^{2}(\partial T)}^{2} \leq c\left(h_{T}^{-1}\|v\|_{L^{2}(T)}^{2}+h_{T}\|\nabla v\|_{L^{2}(T)}^{2}\right) \quad \text { for all } v \in H^{1}(T)
$$

holds, cf. [4]. Take $T \in \mathcal{T}_{h}^{\Gamma}$ and let $\tilde{\Gamma}_{T}=\Gamma_{T}^{(i)}$ be one of the parts of $\Gamma_{T}$ as described in (A2). If $\tilde{\Gamma}_{T}$ coincides with a face of $T$, the result (4.2) immediately follows from (4.3). If this is not the case, the local surface section $\tilde{\Gamma}_{T}$ divides $T$ into two disjoint subdomains $T_{1}, T_{2}$, with $T_{1} \cup T_{2}=T$ and meas $3\left(T_{i}\right)>0$ for $i=1$,2. From (A2) it follows that for $i=1$ or $i=2$ we have $\partial \tilde{\Gamma}_{T} \subset \partial T_{i}$ and $\left(\partial T_{i} \backslash \tilde{\Gamma}_{T}\right) \subset \partial T$. We assume that this holds for $i=1$. Take $x_{0} \in \tilde{\Gamma}_{T}$ such that $n_{h}\left(x_{0}\right)$ exists; we assume that $n_{h}\left(x_{0}\right)$ is outward pointing from $T_{1}$ (otherwise we change the sign). We chose an orthogonal coordinate system $z=\left(z_{1}, z_{2}, z_{3}\right)$ with origin at $x_{0}$ and the third basis vector equal to $n_{h}\left(x_{0}\right)$. The entries of the normal vector $n_{h}(y), y \in \tilde{\Gamma}_{T}$, in the $z$-coordinate system are denoted by $n_{h}(y)=\left(n_{h}^{1}(y), n_{h}^{2}(y), n_{h}^{3}(y)\right)$. Hence, $n_{h}\left(x_{0}\right)=(0,0,1)$. From Assumption 1 (A2) we obtain

$$
\left|n_{h}^{3}(y)-1\right| \leq\left\|n_{h}(y)-n_{h}\left(x_{0}\right)\right\| \leq c_{1} h, \quad \text { for } y \in \tilde{\Gamma}_{T}
$$

Thus there is a constant $c$ such that, for $h$ sufficiently small, $1 \leq n_{h}^{3}(y)^{-1} \leq c$ holds a.e. on $\tilde{\Gamma}_{T}$. For $v \in H^{1}(T)$ we get

$$
\begin{aligned}
2 \int_{T_{1}} v \frac{\partial v}{\partial z_{3}} \mathrm{~d} z & =\int_{T_{1}} \operatorname{div}_{z}\left(\begin{array}{c}
0 \\
0 \\
v^{2}
\end{array}\right) \mathrm{d} z=\int_{\partial T_{1}} n_{T_{1}} \cdot\left(\begin{array}{c}
0 \\
0 \\
v^{2}
\end{array}\right) \mathrm{d} z \\
& =\int_{\tilde{\Gamma}_{T}} n_{h}^{3} v^{2} \mathrm{~d} z+\int_{\partial T_{1} \backslash \tilde{\Gamma}_{T}} n_{T_{1}}^{3} v^{2} \mathrm{~d} z
\end{aligned}
$$

Using $n_{h}^{3}(y)^{-1} \leq c$, we get

$$
\begin{aligned}
\int_{\tilde{\Gamma}_{T}} v^{2} \mathrm{~d} z & \leq c\left(\int_{T_{1}} v \frac{\partial v}{\partial z_{3}} \mathrm{~d} z-\int_{\partial T_{1} \backslash \tilde{\Gamma}_{T}} n_{T}^{3} v^{2} \mathrm{~d} z\right) \\
& \leq c\left(\|v\|_{L^{2}(T)}\|\nabla v\|_{L^{2}(T)}+\|v\|_{L^{2}(\partial T)}^{2}\right) \leq c\left(h_{T}^{-1}\|v\|_{L^{2}(T)}^{2}+h_{T}\|\nabla v\|_{L^{2}(T)}^{2}\right)
\end{aligned}
$$

where in the last inequality we used (4.3). Summing over the parts $\Gamma_{T}^{(i)}, i=1, \ldots, p$, and using that $p$ is uniformly bounded, we obtain the estimate (4.2).

As an easy consequence of the lemmas 3.1 and 4.1 we obtain the following main theorem.

TheOREm 4.2. Let Assumption 1 be satisfied. Let $I_{h}: C\left(\omega_{h}\right) \rightarrow V_{h, m}$ be the nodal interpolation. There exists a constant $c$, independent of $h$ and of how $\Gamma_{h}$ interests $\mathcal{T}_{h}^{\Gamma}$, such that

$$
\begin{aligned}
& \min _{v_{h} \in V_{h, m}^{\Gamma}}\left(\left\|u^{e}-v_{h}\right\|_{L^{2}\left(\Gamma_{h}\right)}+h\left\|\nabla\left(u^{e}-v_{h}\right)\right\|_{L^{2}\left(\Gamma_{h}\right)}\right) \\
& \leq\left\|u^{e}-I_{h} u^{e}\right\|_{L^{2}\left(\Gamma_{h}\right)}+h\left\|\nabla\left(u^{e}-I_{h} u^{e}\right)\right\|_{L^{2}\left(\Gamma_{h}\right)} \leq c h^{m+1}\|u\|_{H^{m+1}(\Gamma)}
\end{aligned}
$$


for all $u \in H^{m+1}(\Gamma)$ holds.

Proof. From $u \in H^{m+1}(\Gamma)$ it follows that $u^{e} \in H^{m+1}\left(\omega_{h}\right)$. Using Lemma 4.1 and standard error bounds for the nodal interpolation $I_{h}$, we obtain, with $v_{h}:=I_{h} u^{e} \in$ $V_{h, m}$ :

$$
\begin{aligned}
& \left\|u^{e}-v_{h}\right\|_{L^{2}\left(\Gamma_{h}\right)}^{2}+h^{2}\left\|\nabla\left(u^{e}-v_{h}\right)\right\|_{L^{2}\left(\Gamma_{h}\right)}^{2} \\
& =\sum_{T \in \mathcal{T}_{h}^{\Gamma}}\left(\left\|u^{e}-v_{h}\right\|_{L^{2}\left(\Gamma_{T}\right)}^{2}+h^{2}\left\|\nabla\left(u^{e}-v_{h}\right)\right\|_{L^{2}\left(\Gamma_{T}\right)}^{2}\right) \\
& \leq c \sum_{T \in \mathcal{T}_{h}^{\Gamma}}\left(h^{-1}\left\|u^{e}-v_{h}\right\|_{L^{2}(T)}^{2}+h\left\|\nabla\left(u^{e}-v_{h}\right)\right\|_{L^{2}(T)}^{2}+h^{3}\left\|\nabla^{2}\left(u^{e}-v_{h}\right)\right\|_{L^{2}(T)}^{2}\right) \\
& \leq c \sum_{T \in \mathcal{T}_{h}^{\Gamma}} h^{2 m+1}\left\|u^{e}\right\|_{H^{m+1}(T)}^{2}=c h^{2 m+1}\left\|u^{e}\right\|_{H^{m+1}\left(\omega_{h}\right)}^{2} .
\end{aligned}
$$

Using Assumption 1 (A1) and (3.8) with $\delta=c_{0} h$ we get $\left\|u^{e}\right\|_{H^{m+1}\left(\omega_{h}\right)}^{2} \leq c h\|u\|_{H^{m+1}(\Gamma)}^{2}$, which completes the proof.

From the result in this theorem we conclude that for the trace space $V_{h, m}^{\Gamma}$ we have optimal approximation error bounds under (very) mild conditions on the approximate surface $\Gamma_{h}$. If $\Gamma$ and the exact solution $u$ are sufficiently smooth, we obtain an $h^{m+1}$ bound as in (4.4) (for finite elements of degree $m$ ), provided Assumption 1 is satisfied. The latter essentially only requires the accuracy $\operatorname{dist}\left(\Gamma_{h}, \Gamma\right) \leq c h$ for the approximate surface.

5. Finite element error bounds. In this section we prove optimal discretization error bounds both in the $H^{1}\left(\Gamma_{h}\right)$ and the $L^{2}\left(\Gamma_{h}\right)$ norm. For the discrete problem (2.5) such bounds for $m=1$ (piecewise linear finite elements) are derived in [7]. For the discrete problem (2.6) these error bounds for $m=1$ are derived in [24]. In both references it is assumed that $\Gamma_{h}$ is a piecewise planar approximation of $\Gamma$ with $\operatorname{dist}\left(\Gamma_{h}, \Gamma\right) \leq c h^{2}$. In this section we consider a more general setting with $m \geq 1$ and more general approximate surfaces $\Gamma_{h}$. Furthermore, we present the error analysis of the two discretizations in one unified setting, which reveals the main (theoretical) differences between the two methods.

In the analysis we need one further assumption, which quantifies the quality of $\Gamma_{h}$ as an approximation of $\Gamma$ ("geometric error").

Assumption 2. We assume that $\Gamma_{h} \subset U_{\delta_{0}}$ is a Lipschitz surface without boundary and that the projection $p: \Gamma_{h} \rightarrow \Gamma$ is a bijection. The corresponding unit normal field is defined a.e. on $\Gamma_{h}$ and denoted by $n_{h}$. We assume that the following holds, for a $k \geq 1$ :

$$
\begin{aligned}
\|d\|_{L^{\infty}\left(\Gamma_{h}\right)} & \leq c h^{k+1}, \\
\left\|n-n_{h}\right\|_{L^{\infty}\left(\Gamma_{h}\right)} & \leq c h^{k} .
\end{aligned}
$$

These are the key assumptions we need in the analysis below. There is one further assumption we introduce. On each $\Gamma_{h}$ there holds a Poincare inequality with a constant $c=c(h)$. We assume that this constant is uniform w.r.t. $h$, i.e., we assume that there exists $c$, independent of $h$, such that

$$
\|v\|_{L^{2}\left(\Gamma_{h}\right)} \leq c\left\|\nabla_{\Gamma_{h}} v\right\|_{L^{2}\left(\Gamma_{h}\right)} \text { for all } v \in H^{1}\left(\Gamma_{h}\right) / \mathbb{R} .
$$


REMARK 2. We discuss cases in which the assumptions (5.1)-(5.2) are satisfied. Clearly, if $\Gamma_{h}=\Gamma$ there is no geometric error, i.e. these assumptions are fulfilled with $k=\infty$. Consider the case in which $\Gamma$ is the zero level of a smooth level set function $\phi$ and $\phi_{h}$ is a finite element approximation of $\phi$, on the triangulation $\mathcal{T}_{h}$. Let $\Gamma_{h}$ be the zero level of $\phi_{h}$. If $\left\|\phi-\phi_{h}\right\|_{L^{\infty}\left(\omega_{h}\right)}+h\left\|\nabla\left(\phi-\phi_{h}\right)\right\|_{L^{\infty}\left(\omega_{h}\right)} \leq c h^{k+1}$ holds, then the conditions (5.1)-(5.2) are satisfied. In [8] a method for constructing polynomial approximations to $\Gamma$ is presented that satisfies the conditions (5.1)-(5.2) (cf. Proposition 2.3 in [8]). In that method the exact distance function to $\Gamma$ is needed.

We define the following projections

$$
P_{h}(x)=I-n_{h}(x) n_{h}(x)^{T}, \quad \tilde{P}_{h}(x)=I-n_{h}(x) n(x)^{T} /\left(n_{h}(x)^{T} n(x)\right), \quad x \in \Gamma_{h} .
$$

We collect a few results from [9]. The surface gradient of $u \in H^{1}(\Gamma)$ can be represented in terms of $\nabla_{\Gamma_{h}} u^{e}$ as follows:

$$
\nabla_{\Gamma} u(p(x))=(I-d(x) H(x))^{-1} \tilde{P}_{h}(x) \nabla_{\Gamma_{h}} u^{e}(x) \quad \text { a.e. on } \Gamma_{h} .
$$

For $x \in \Gamma_{h}$ define

$$
\mu_{h}(x)=\left(1-d(x) \kappa_{1}(x)\right)\left(1-d(x) \kappa_{1}(x)\right) n(x)^{T} n_{h}(x) .
$$

The integral transformation formula

$$
\mu_{h}(x) \mathrm{d} s_{h}(x)=\mathrm{d} s(p(x)), \quad x \in \Gamma_{h},
$$

holds, where $\mathrm{d} s_{h}(x)$ and $\mathrm{d} s(p(x))$ are the surface measures on $\Gamma_{h}$ and $\Gamma$, respectively. From $\left\|n(x)-n_{h}(x)\right\|^{2}=2\left(1-n(x)^{T} n_{h}(x)\right)$, and Assumption 2 we obtain

$$
\left\|1-\mu_{h}\right\|_{L^{\infty}\left(\Gamma_{h}\right)} \leq c h^{k+1},
$$

with a constant $c$ independent of $h$. Using relation (5.4) and (5.5) we obtain

$$
\begin{aligned}
\int_{\Gamma} \nabla_{\Gamma} u \cdot \nabla_{\Gamma} v \mathrm{~d} \mathbf{s} & =\int_{\Gamma_{h}} A_{h} \nabla_{\Gamma_{h}} u^{e} \cdot \nabla_{\Gamma_{h}} v^{e} \mathrm{~d} \mathbf{s}_{h} \text { for all } u, v \in H^{1}(\Gamma), \\
\text { with } A_{h}(x) & =\mu_{h}(x) \tilde{P}_{h}(x)(I-d(x) H(x))^{-2} \tilde{P}_{h}(x) .
\end{aligned}
$$

We introduce a compact notation for the bilinear forms used in (2.5), (2.6):

$$
a_{h}\left(u_{h}, v_{h}\right):=\int_{\Gamma_{h}} \nabla u_{h} \cdot \nabla v_{h} \mathrm{~d} s_{h}, \quad a_{h}^{\Gamma}\left(u_{h}, v_{h}\right):=\int_{\Gamma_{h}} \nabla_{\Gamma_{h}} u_{h} \cdot \nabla_{\Gamma_{h}} v_{h} \mathrm{~d} s_{h} .
$$

Furthermore, for the data error we introduce the notation

$$
\delta_{f}:=f_{h}-\mu_{h} f^{e} .
$$

We now derive approximate Galerkin orthogonality relations for the discrete problems.

LEMma 5.1. Let $u$ be the solution of the Laplace-Beltrami equation (2.2) and $u_{h}, u_{h}^{\Gamma} \in V_{h, m}^{\Gamma}$ the solutions of the discrete problems (2.5) and (2.6), respectively. Define $\hat{A}_{h}:=P_{h} A_{h} P_{h}$, with $A_{h}$ as in (5.8). The following holds:

$$
\begin{aligned}
a_{h}\left(u^{e}-u_{h}, v_{h}\right) & =F_{h}\left(v_{h}\right) \quad \text { for all } v_{h} \in V_{h, m}^{\Gamma}, \\
\text { with } F_{h}\left(v_{h}\right) & :=\int_{\Gamma_{h}}\left(I-\hat{A}_{h}\right) \nabla u^{e} \cdot \nabla v_{h} \mathrm{~d} s_{h}-\int_{\Gamma_{h}} \delta_{f} v_{h} \mathrm{~d} s_{h} . \\
a_{h}^{\Gamma}\left(u^{e}-u_{h}^{\Gamma}, v_{h}\right) & =F_{h}^{\Gamma}\left(v_{h}\right) \quad \text { for all } v_{h} \in V_{h, m}^{\Gamma}, \\
\text { with } F_{h}^{\Gamma}\left(v_{h}\right) & :=\int_{\Gamma_{h}}\left(P_{h}-\hat{A}_{h}\right) \nabla u^{e} \cdot \nabla v_{h} \mathrm{~d} s_{h}-\int_{\Gamma_{h}} \delta_{f} v_{h} \mathrm{~d} s_{h} .
\end{aligned}
$$


Proof. A function $v_{h}$ on $\Gamma_{h}$ can be lifted on $\Gamma$ by defining $v_{h}^{l}(p(x)):=v_{h}(x)$. From the definition of the discrete problem (2.5) and the transformation rule (5.7) we get

$$
\begin{aligned}
& a_{h}\left(u_{h}, v_{h}\right)=\int_{\Gamma_{h}} f_{h} v_{h} \mathrm{~d} s_{h}=\int_{\Gamma} f v_{h}^{l} \mathrm{~d} s+\int_{\Gamma_{h}} \delta_{f} v_{h} \mathrm{~d} s_{h} \\
& =\int_{\Gamma} \nabla_{\Gamma} u \cdot \nabla_{\Gamma} v_{h}^{l} \mathrm{~d} s+\int_{\Gamma_{h}} \delta_{f} v_{h} \mathrm{~d} s_{h}=\int_{\Gamma_{h}} A_{h} \nabla_{\Gamma_{h}} u^{e} \cdot \nabla_{\Gamma_{h}} v_{h} \mathrm{~d} s+\int_{\Gamma_{h}} \delta_{f} v_{h} \mathrm{~d} s_{h} \\
& =\int_{\Gamma_{h}} \hat{A}_{h} \nabla u^{e} \cdot \nabla v_{h} \mathrm{~d} s+\int_{\Gamma_{h}} \delta_{f} v_{h} \mathrm{~d} s_{h}
\end{aligned}
$$

where in the last inequality we used that $\nabla_{\Gamma_{h}} v_{h}=P_{h} \nabla v_{h}$. Combining this with $a_{h}\left(u^{e}, v_{h}\right)=\int_{\Gamma_{h}} \nabla u^{e} \cdot \nabla v_{h} \mathrm{~d} s_{h}$ we get the result in (5.9). Similar arguments can be used to derive (5.10):

$$
\begin{aligned}
& a_{h}^{\Gamma}\left(u_{h}^{\Gamma}, v_{h}\right)=\int_{\Gamma_{h}} \nabla_{\Gamma_{h}} u_{h}^{\Gamma} \cdot \nabla_{\Gamma_{h}} v_{h} \mathrm{~d} \mathbf{s}_{h}=\int_{\Gamma} f v_{h}^{l} \mathrm{~d} s+\int_{\Gamma_{h}} \delta_{f} v_{h} \mathrm{~d} s_{h} \\
& =\int_{\Gamma_{h}} \hat{A}_{h} \nabla u^{e} \cdot \nabla v_{h} \mathrm{~d} s+\int_{\Gamma_{h}} \delta_{f} v_{h} \mathrm{~d} s_{h} .
\end{aligned}
$$

We combine this with $a_{h}^{\Gamma}\left(u^{e}, v_{h}\right)=\int_{\Gamma_{h}} P_{h} \nabla u^{e} \cdot \nabla v_{h} \mathrm{~d} s_{h}$ and thus get (5.10).

Note that the only difference between the perturbation terms $F_{h}$ and $F_{h}^{\Gamma}$ in (5.9) and (5.10) is in the matrices $I-\hat{A}_{h}$ and $P_{h}-\hat{A}_{h}$. We derive bounds for the perturbation terms $F_{h}$ and $F_{h}^{\Gamma}$. We need some additional notation, namely $H^{2}(\Gamma)^{e}:=\left\{v^{e} \mid v \in\right.$ $\left.H^{2}(\Gamma)\right\}$.

LEMma 5.2. Let Assumption 2 be fulfilled and assume that the data error satisfies $\left\|\delta_{f}\right\|_{L^{2}\left(\Gamma_{h}\right)} \leq c h^{k+s}\|f\|_{L^{2}(\Gamma)}$ for an $s \in[0,1]$. The following holds, with constants $c$ independent of $h$ :

$$
\begin{aligned}
\left|F_{h}(v)\right| & \leq c h^{k}\|f\|_{L^{2}(\Gamma)}\left(\|v\|_{L^{2}\left(\Gamma_{h}\right)}+\|\nabla v\|_{L^{2}\left(\Gamma_{h}\right)}\right) \quad \forall v \in V_{h, m}^{\Gamma}+H^{2}(\Gamma)^{e} \\
\left|F_{h}^{\Gamma}(v)\right| & \leq c h^{k+s}\|f\|_{L^{2}(\Gamma)}\left(\|v\|_{L^{2}\left(\Gamma_{h}\right)}+\left\|\nabla_{\Gamma_{h}} v\right\|_{L^{2}\left(\Gamma_{h}\right)}\right) \forall v \in V_{h, m}^{\Gamma}+H^{2}(\Gamma)^{e}, \\
\left|F_{h}\left(v^{e}\right)\right| & \leq c h^{k+s}\|f\|_{L^{2}(\Gamma)}\left(\|v\|_{L^{2}(\Gamma)}+\left\|\nabla_{\Gamma} v\right\|_{L^{2}(\Gamma)}\right) \quad \forall v \in H^{2}(\Gamma) .
\end{aligned}
$$

Proof. For the second term in $F_{h}(v)$ and $F_{h}^{\Gamma}(v)$ we get

$$
\left|\int_{\Gamma_{h}} \delta_{f} v \mathrm{~d} s_{h}\right| \leq\left\|\delta_{f}\right\|_{L^{2}\left(\Gamma_{h}\right)}\|v\|_{L^{2}\left(\Gamma_{h}\right)} \leq c h^{k+s}\|f\|_{L^{2}(\Gamma)}\|v\|_{L^{2}\left(\Gamma_{h}\right)} .
$$

Below we delete the argument $x \in \Gamma_{h}$ in the notation. Using (3.4), $P(p(x))=P(x)$ and $H P=P H$ we get

$$
\left(I-\hat{A}_{h}\right) \nabla u^{e}=\left(I-\hat{A}_{h}\right) P(I-d H) \nabla_{\Gamma} u(p(x)) .
$$

We combine $\hat{A}_{h}=P_{h} A_{h} P_{h}$ with the definition of $A_{h}$ and with (5.1), (5.6), $P_{h} \tilde{P}_{h}=P_{h}$ and obtain

$$
\left\|\hat{A}_{h}-P_{h}\right\|_{L^{\infty}\left(\Gamma_{h}\right)} \leq c h^{k+1}
$$


Hence, using (5.2) yields

$$
\begin{aligned}
\left\|\left(I-\hat{A}_{h}\right) P\right\|_{L^{\infty}\left(\Gamma_{h}\right)} & \leq\left\|\left(I-P_{h}\right) P\right\|_{L^{\infty}\left(\Gamma_{h}\right)}+c h^{k+1} \\
& \leq\left\|P-P_{h}\right\|_{L^{\infty}\left(\Gamma_{h}\right)}+c h^{k+1} \leq c h^{k} .
\end{aligned}
$$

With the result in (5.15) we thus obtain

$$
\begin{aligned}
& \left|\int_{\Gamma_{h}}\left(I-\hat{A}_{h}\right) \nabla u^{e} \cdot \nabla v \mathrm{~d} s_{h}\right| \leq c h^{k}\left\|\nabla_{\Gamma} u(p(\cdot))\right\|_{L^{2}\left(\Gamma_{h}\right)}\|\nabla v\|_{L^{2}\left(\Gamma_{h}\right)} \\
& \leq c h^{k}\left\|\nabla_{\Gamma} u\right\|_{L^{2}(\Gamma)}\|\nabla v\|_{L^{2}\left(\Gamma_{h}\right)} \leq c h^{k}\|f\|_{L^{2}(\Gamma)}\|\nabla v\|_{L^{2}\left(\Gamma_{h}\right)},
\end{aligned}
$$

and combining this with the result in (5.14) completes the proof of (5.11). In the definition of $F_{h}^{\Gamma}(v)$ we have the matrix $P_{h}-\hat{A}_{h}$, instead of $I-\hat{A}_{h}$. For the former we have, cf. (5.16), $\left\|\hat{A}_{h}-P_{h}\right\|_{L^{\infty}\left(\Gamma_{h}\right)} \leq c h^{k+1}$. Furthermore we have

$$
\left(P_{h}-\hat{A}_{h}\right) \nabla u^{e} \cdot \nabla v=P_{h}\left(P_{h}-\hat{A}_{h}\right) \nabla u^{e} \cdot \nabla v=\left(P_{h}-\hat{A}_{h}\right) \nabla u^{e} \cdot \nabla_{\Gamma_{h}} v .
$$

Similarly as in (5.18) we obtain

$$
\begin{aligned}
\left|\int_{\Gamma_{h}}\left(P_{h}-\hat{A}_{h}\right) \nabla u^{e} \cdot \nabla v \mathrm{~d} s_{h}\right| & \leq c h^{k+1}\left\|\nabla_{\Gamma} u(p(\cdot))\right\|_{L^{2}\left(\Gamma_{h}\right)}\left\|\nabla_{\Gamma_{h}} v\right\|_{L^{2}\left(\Gamma_{h}\right)} \\
& \leq c h^{k+1}\|f\|_{L^{2}(\Gamma)}\left\|\nabla_{\Gamma_{h}} v\right\|_{L^{2}\left(\Gamma_{h}\right)},
\end{aligned}
$$

and combining this with the result in (5.14) we get the bound (5.12). We finally consider the estimate (5.13). We use (3.4) and thus get, cf. (5.15),

$$
\left(I-\hat{A}_{h}\right) \nabla u^{e} \cdot \nabla v^{e}=\left[(I-d H) P\left(I-\hat{A}_{h}\right) P(I-d H)\right] \nabla_{\Gamma} u(p(x)) \cdot \nabla_{\Gamma} v(p(x)) .
$$

For the matrix in the square brackets we have, cf. (5.16),

$$
\left\|(I-d H) P\left(I-\hat{A}_{h}\right) P(I-d H)\right\|_{L^{\infty}\left(\Gamma_{h}\right)} \leq\left\|P\left(I-P_{h}\right) P\right\|_{L^{\infty}\left(\Gamma_{h}\right)}+c h^{k+1} .
$$

Using $P\left(I-P_{h}\right) P=P n_{h} n_{h}^{T} P=\left(P-P_{h}\right) n_{h} n_{h}^{T}\left(P-P_{h}\right)$ and (5.2) we obtain $\left\|P\left(I-P_{h}\right) P\right\|_{L^{\infty}\left(\Gamma_{h}\right)} \leq c h^{2 k}$. From this is follows that the norm of the matrix in the square brackets is bounded by $c h^{k+1}$. Using similar arguments as in the derivation of (5.12) above we then obtain the bound (5.13).

REMARK 3. We comment on the data error $\left\|\delta_{f}\right\|_{L^{2}\left(\Gamma_{h}\right)}$, with $\delta_{f}=f_{h}-\mu_{h} f^{e}$. For the choice $f_{h}=f^{e}-\frac{1}{\left|\Gamma_{h}\right|} \int_{\Gamma_{h}} f^{e} \mathrm{~d} s_{h}$, which in practice often can not be realized, we obtain, using (5.6), the data error bound $\left\|\delta_{f}\right\|_{L^{2}\left(\Gamma_{h}\right)} \leq c h^{k+1}\|f\|_{L^{2}(\Gamma)}$. Another, more feasible, possibility arises if we assume that $f$ is a smooth function on $U_{\delta_{0}}$. As extension one can then use

$$
f_{h}(x)=f(x)-c_{f}, \quad c_{f}:=\frac{1}{\left|\Gamma_{h}\right|} \int_{\Gamma_{h}} f \mathrm{~d} s_{h} .
$$

Using $\int_{\Gamma} f \mathrm{~d} s=0,(5.1),(5.6)$ and a Taylor expansion we get $\left|c_{f}\right| \leq c h^{k+1}\|f\|_{H_{\infty}^{1}\left(U_{\delta_{0}}\right)}$ and $\left\|f-\mu_{h} f^{e}\right\|_{L^{2}\left(\Gamma_{h}\right)} \leq c h^{k+1}\|f\|_{H_{\infty}^{1}\left(U_{\delta_{0}}\right)}$. Hence, a data error bound $\left\|\delta_{f}\right\|_{L^{2}\left(\Gamma_{h}\right)} \leq$ $\hat{c} h^{k+1}\|f\|_{L^{2}(\Gamma)}$ with $\hat{c}=\hat{c}(f)=c\|f\|_{H_{\infty}^{1}\left(U_{\delta_{0}}\right)}\|f\|_{L^{2}(\Gamma)}^{-1}$ and a constant $c$ independent of $f$. Hence, in problems with smooth data it is realistic to assume that the condition on the data error in Lemma 5.2 is satisfied with $s=1$. In less regular situations, $s<1$ 
may be more realistic.

Note that for $s>0$ the bound on $F_{h}^{\Gamma}$ in (5.12) is of higher order in $h$ than the one for $F_{h}$ in (5.11). This difference is reflected in the discretization error bounds derived below. For $F_{h}\left(v^{e}\right)$ the higher order bound in (5.13) is obtained by using the special structure of $v^{e}$ (namely constant in normal direction). The latter bound is used in the proof of the $L^{2}$-error bound in Theorem 5.4 below.

Theorem 5.3. Let the Assumptions 1 and 2 be fulfilled. Assume that the data error satisfies $\left\|\delta_{f}\right\|_{L^{2}\left(\Gamma_{h}\right)} \leq c h^{k+s}\|f\|_{L^{2}(\Gamma)}$ for an $s \in[0,1]$. Let $u_{h}$ and $u_{h}^{\Gamma}$ be the solutions of the discrete problems (2.5) and (2.6), respectively. The following error bounds hold, with a constant $c$ independent of $h$ and $f$ :

$$
\begin{aligned}
\left\|\nabla\left(u^{e}-u_{h}\right)\right\|_{L^{2}\left(\Gamma_{h}\right)} & \leq c\left(h^{m}\|u\|_{H^{m+1}(\Gamma)}+h^{k}\|f\|_{L^{2}(\Gamma)}\right) \\
\left\|\nabla_{\Gamma_{h}}\left(u^{e}-u_{h}^{\Gamma}\right)\right\|_{L^{2}\left(\Gamma_{h}\right)} & \leq c\left(h^{m}\|u\|_{H^{m+1}(\Gamma)}+h^{k+s}\|f\|_{L^{2}(\Gamma)}\right) .
\end{aligned}
$$

Proof. Define $e_{h}:=u^{e}-u_{h}$ and $\psi_{h}:=\left(I_{h} u^{e}\right)_{\mid \Gamma_{h}} \in V_{h, m}^{\Gamma}$. We consider the splitting

$$
\left\|\nabla e_{h}\right\|_{L^{2}\left(\Gamma_{h}\right)}^{2}=a_{h}\left(e_{h}, e_{h}\right)=a_{h}\left(e_{h}, u^{e}-\psi_{h}\right)+F_{h}\left(\psi_{h}-u^{e}\right)+F_{h}\left(e_{h}\right) .
$$

For the first two terms on the right-hand side we use (5.11) and the interpolation error bounds of Theorem 4.2 and thus get

$$
\begin{aligned}
& a_{h}\left(e_{h}, u^{e}-\psi_{h}\right)+F_{h}\left(\psi_{h}-u^{e}\right) \leq c\left(\left\|\nabla e_{h}\right\|_{L^{2}\left(\Gamma_{h}\right)}+h^{k}\|f\|_{L^{2}\left(\Gamma_{h}\right)}\right) h^{m}\|u\|_{H^{m+1}(\Gamma)} \\
& \leq \frac{1}{4}\left\|\nabla e_{h}\right\|_{L^{2}\left(\Gamma_{h}\right)}^{2}+c h^{2 m}\|u\|_{H^{m+1}(\Gamma)}^{2}+c h^{2 k}\|f\|_{L^{2}\left(\Gamma_{h}\right)}^{2} .
\end{aligned}
$$

For the third term we need the Poincare inequality (5.3). Define $c_{u}=\int_{\Gamma_{h}} u^{e} \mathrm{~d} s_{h}$. Using $\int_{\Gamma} u \mathrm{~d} s=0$ and (5.6) we get $\left|c_{u}\right| \leq c h^{k+1}\|u\|_{L^{2}(\Gamma)} \leq c h^{k+1}\|f\|_{L^{2}(\Gamma)}$. Note that $\int_{\Gamma_{h}} e_{h}-c_{u} \mathrm{~d} s_{h}=0$ holds, hence with the Poincare inequality we obtain

$$
\begin{aligned}
\left\|e_{h}\right\|_{L^{2}\left(\Gamma_{h}\right)} & \leq\left\|e_{h}-c_{u}\right\|_{L^{2}\left(\Gamma_{h}\right)}+c h^{k+1}\|f\|_{L^{2}(\Gamma)} \\
& \leq c\left\|\nabla_{\Gamma_{h}} e_{h}\right\|_{L^{2}\left(\Gamma_{h}\right)}+c h^{k+1}\|f\|_{L^{2}(\Gamma)} \leq c\left\|\nabla e_{h}\right\|_{L^{2}\left(\Gamma_{h}\right)}+c h^{k+1}\|f\|_{L^{2}(\Gamma)},
\end{aligned}
$$

and using this in the estimate (5.11) yields

$$
F_{h}\left(e_{h}\right) \leq c h^{k}\|f\|_{L^{2}(\Gamma)}\left(\left\|\nabla e_{h}\right\|_{L^{2}\left(\Gamma_{h}\right)}+h^{k+1}\|f\|_{L^{2}(\Gamma)}\right) \leq \frac{1}{4}\left\|\nabla e_{h}\right\|_{L^{2}\left(\Gamma_{h}\right)}^{2}+c h^{2 k}\|f\|_{L^{2}(\Gamma)}^{2} .
$$

Combining this with the result in (5.21) proves the bound in (5.19). The result in (5.20) follows with very similar arguments. Define $e_{h}^{\Gamma}=u^{e}-u_{h}^{\Gamma}$, and consider the splitting

$$
\left\|\nabla_{\Gamma_{h}} e_{h}^{\Gamma}\right\|_{L^{2}\left(\Gamma_{h}\right)}^{2}=a_{h}^{\Gamma}\left(e_{h}^{\Gamma}, e_{h}^{\Gamma}\right)=a_{h}^{\Gamma}\left(e_{h}^{\Gamma}, u^{e}-\psi_{h}\right)+F_{h}^{\Gamma}\left(\psi_{h}-u^{e}\right)+F_{h}^{\Gamma}\left(e_{h}^{\Gamma}\right) .
$$

Note that $\left\|\nabla_{\Gamma_{h}}\left(u^{e}-\psi_{h}\right)\right\|_{L^{2}\left(\Gamma_{h}\right)} \leq\left\|\nabla\left(u^{e}-\psi_{h}\right)\right\|_{L^{2}\left(\Gamma_{h}\right)}$, hence for bounding the interpolation error we can use Theorem 4.2. We can repeat the arguments used above. Since in the bound for $F_{h}^{\Gamma}(v)$ in (5.12) we have a term $h^{k+s}$ (instead of $h^{k}$ ) we get the factor $h^{k+s}$ in the bound (5.20).

The result in this theorem yields optimal $H^{1}$-error bounds for both methods, also for the case of higher order finite elements $(m \geq 2)$. Of course, this optimal bound is 
obtained only if the approximation error term, which is of order $h^{m}$, is not dominated by the geometric error term, which is of order $h^{k}$ and $h^{k+s}$, respectively. Assume $s=1$, cf. Remark 3. For the case $k=m$ (which typically holds in case of linear finite elements, i.e., $m=1$ ), we see that for the method in (2.5) the geometric error is of the same order as the approximation error, whereas for the method (2.6) the geometric error is of higher order. For the method in (2.6) and $m \geq 2$ we get the optimal order of convergence $h^{m}$ even if we only have $k=m-1$. The method (2.5) does not have this property.

We apply a duality argument to obtain an $L^{2}\left(\Gamma_{h}\right)$-error bound. In this analysis the estimate (5.13) is used.

TheOrem 5.4. Let the Assumptions 1 and 2 be fulfilled. Assume that the data error satisfies $\left\|\delta_{f}\right\|_{L^{2}\left(\Gamma_{h}\right)} \leq c h^{k+s}\|f\|_{L^{2}(\Gamma)}$ for an $s \in[0,1]$. Let $u_{h}$ and $u_{h}^{\Gamma}$ be the solutions of the discrete problems (2.5) and (2.6), respectively. The following error bounds hold, with a constant $c$ independent of $h$ and $f$ :

$$
\begin{aligned}
& \left\|u^{e}-u_{h}\right\|_{L^{2}\left(\Gamma_{h}\right)} \leq c\left(h^{m+1}\|u\|_{H^{m+1}(\Gamma)}+h^{k+s}\|f\|_{L^{2}(\Gamma)}\right) \\
& \left\|u^{e}-u_{h}^{\Gamma}\right\|_{L^{2}\left(\Gamma_{h}\right)} \leq c\left(h^{m+1}\|u\|_{H^{m+1}(\Gamma)}+h^{k+s}\|f\|_{L^{2}(\Gamma)}\right) .
\end{aligned}
$$

Proof. Denote $e_{h}:=u^{e}-u_{h}$ and let $e_{h}^{l}$ be the lift of $e_{h \mid \Gamma_{h}}$ on $\Gamma$ and $c_{e}:=\int_{\Gamma} e_{h}^{l} \mathrm{~d} s$. Consider the problem: Find $w \in H^{1}(\Gamma)$ with $\int_{\Gamma} w \mathrm{~d} s=0$ such that

$$
\int_{\Gamma} \nabla_{\Gamma} w \cdot \nabla_{\Gamma} v \mathrm{~d} s=\int_{\Gamma}\left(e_{h}^{l}-c_{e}\right) v \mathrm{~d} s \quad \text { for all } v \in H^{1}(\Gamma) .
$$

The solution $w$ satisfies $w \in H^{2}(\Gamma)$ and $\|w\|_{H^{2}(\Gamma)} \leq c\left\|e_{h}^{l}\right\|_{L^{2}(\Gamma) / \mathbb{R}}$ with $\left\|e_{h}^{l}\right\|_{L^{2}(\Gamma) / \mathbb{R}}:=$ $\left\|e_{h}^{l}-c_{e}\right\|_{L^{2}(\Gamma)}$. We take $\psi_{h}=I_{h} w^{e} \in V_{h, m}$ and with $\hat{A}_{h}=P_{h} A_{h} P_{h}$ we obtain

$$
\begin{aligned}
& \left\|e_{h}^{l}\right\|_{L^{2}(\Gamma) / \mathbb{R}}^{2}=\int_{\Gamma} \nabla_{\Gamma} w \cdot \nabla_{\Gamma}\left(e_{h}^{l}-c_{e}\right) \mathrm{d} s=\int_{\Gamma} \nabla_{\Gamma} w \nabla_{\Gamma} e_{h}^{l} \mathrm{~d} s \\
& =\int_{\Gamma_{h}} A_{h} \nabla_{\Gamma_{h}} e_{h} \cdot \nabla_{\Gamma_{h}} w^{e} \mathrm{~d} s_{h}=\int_{\Gamma_{h}} \nabla e_{h} \cdot \nabla w^{e} \mathrm{~d} s_{h}+\int_{\Gamma_{h}} \nabla e_{h} \cdot\left(\hat{A}_{h}-I\right) \nabla w^{e} \mathrm{~d} s_{h} \\
& =a_{h}\left(e_{h}, w^{e}-\psi_{h}\right)+F_{h}\left(\psi_{h}-w^{e}\right)+F_{h}\left(w^{e}\right)+\int_{\Gamma_{h}} \nabla e_{h} \cdot\left(\hat{A}_{h}-I\right) \nabla w^{e} \mathrm{~d} s_{h} .
\end{aligned}
$$

We consider the four terms in (5.25). Using the interpolation error bound in Theorem 4.2 (with $m=1$ ), the error bound in Theorem 5.3 and $\|w\|_{H^{2}(\Gamma)} \leq c\left\|e_{h}^{l}\right\|_{L^{2}(\Gamma) / \mathbb{R}}$ we get

$$
a_{h}\left(e_{h}, w^{e}-\psi_{h}\right) \leq c\left(h^{m+1}\|u\|_{H^{m+1}(\Gamma)}+h^{k+1}\|f\|_{L^{2}(\Gamma)}\right)\left\|e_{h}^{l}\right\|_{L^{2}(\Gamma) / \mathbb{R}} .
$$

For the second term we use (5.11) and the interpolation error bound (with $m=1$ ), which yields

$$
F_{h}\left(\psi_{h}-w^{e}\right) \leq c h^{k+1}\|f\|_{L^{2}(\Gamma)}\left\|e_{h}^{l}\right\|_{L^{2}(\Gamma) / \mathbb{R}}
$$

For the third term we use (5.13) and get

$$
F_{h}\left(w^{e}\right) \leq c h^{k+s}\|f\|_{L^{2}(\Gamma)}\|w\|_{H^{1}(\Gamma)} \leq c h^{k+s}\|f\|_{L^{2}(\Gamma)}\left\|e_{h}^{l}\right\|_{L^{2}(\Gamma) / \mathbb{R}} .
$$


For the last term we use (5.15) (with $u$ replaced by $w$ ) and (5.17), which yields

$$
\begin{aligned}
\int_{\Gamma_{h}} \nabla e_{h} \cdot\left(\hat{A}_{h}-I\right) \nabla w^{e} \mathrm{~d} s_{h} & \leq c h^{k}\left\|\nabla e_{h}\right\|_{L^{2}\left(\Gamma_{h}\right)}\left\|\nabla_{\Gamma} w\right\|_{L^{2}(\Gamma)} \\
& \leq c\left(h^{m+k}\|u\|_{H^{m+1}(\Gamma)}+h^{2 k}\|f\|_{L^{2}(\Gamma)}\right)\left\|e_{h}^{l}\right\|_{L^{2}(\Gamma) / \mathbb{R}} .
\end{aligned}
$$

Using these bounds in (5.25) yields

$$
\left\|e_{h}^{l}\right\|_{L^{2}(\Gamma) / \mathbb{R}} \leq c\left(h^{m+1}\|u\|_{H^{m+1}(\Gamma)}+h^{k+s}\|f\|_{L^{2}(\Gamma)}\right) .
$$

Now note that

$$
\left|c_{e}\right|=\left|\int_{\Gamma} u-u_{h}^{l} \mathrm{~d} s\right|=\left|\int_{\Gamma} u_{h}^{l} \mathrm{~d} s\right|=\left|\int_{\Gamma_{h}}\left(\mu_{h}-1\right) u_{h} \mathrm{~d} s_{h}\right| \leq c h^{k+1}\|f\|_{L^{2}(\Gamma)},
$$

and thus

$$
\left\|e_{h}\right\|_{L^{2}\left(\Gamma_{h}\right)} \leq c\left\|e_{h}^{l}\right\|_{L^{2}(\Gamma)} \leq c\left\|e_{h}^{l}\right\|_{L^{2}(\Gamma) / \mathbb{R}}+c h^{k+1}\|f\|_{L^{2}(\Gamma)},
$$

and combining this with (5.27) completes the proof of (5.22). The result (5.23) can be proved with very similar arguments. A proof of (5.23) for $m=k=1$ is given in [24].

Note that the bounds in (5.22) and (5.23) are the same. We have an optimal error if $k+s \geq m+1$ holds. If we have an optimal data approximation error, i.e. $s=1$ cf. Remark 3, we need $k \geq m$ to obtain an optimal $L^{2}$-error bound of order $h^{m+1}$. Inspection of the proof above shows that the factor $h^{k+s}$ in (5.22) originates (only) from the estimate (5.26). All other geometric error terms are of order $h^{k+1}$. In the proof of (5.23) the term $F_{h}^{\Gamma}\left(w^{e}\right)$ has to be bounded. For this the estimate (5.12) is used. Inspection of the proof of the latter estimate reveals that the factor $h^{k+s}$ in the bound in (5.12) can not be improved if we use the special choice $v=w^{e}$. Thus in both error bounds, (5.22) and (5.23), we get the same geometric error term of order $h^{k+s}$.

6. Conditioning of the stiffness matrix. In this section we address linear algebra aspects of the discretizations in (2.5) and (2.6). The discrete solution is determined by using the standard nodal basis of the (outer) finite element space $V_{h, m}$. This nodal basis and the corresponding nodes are denoted by $\left\{\phi_{i}\right\}_{1 \leq i \leq N}$ and $\left\{x_{i}\right\}_{1 \leq i \leq N}$, respectively. Hence, $V_{h, m}=\operatorname{span}\left\{\left(\phi_{i}\right)_{\mid \omega_{h}} \mid 1 \leq i \leq N\right\}$, and $\operatorname{dim}\left(V_{h, m}\right)=$ $N$. By construction we have $\operatorname{span}\left\{\left(\phi_{i}\right)_{\mid \Gamma_{h}} \mid 1 \leq i \leq N\right\}=V_{h, m}^{\Gamma}$. Related to the linear algebra, a key point is that in general the $\left\{\left(\phi_{i}\right)_{\mid \Gamma_{h}}\right\}_{1 \leq i \leq N}$ are not independent, hence these do not form a basis of the trace space $V_{h, m}^{\Gamma}$. This can be illustrated by simple examples, cf. [23]. The representation $v_{h}=\sum_{i=1}^{N} V_{i} \phi_{i}, v_{h} \in V_{h, m}$, induces the isomorphism $v_{h} \rightarrow V:=\left(V_{i}\right)_{1 \leq i \leq N} \in \mathbb{R}^{N}$. The vector corresponding to $w_{h} \in V_{h, m}$ is deneoted by $W$. We introduce the mass and stiffness matrices:

$$
\begin{aligned}
\langle M V, W\rangle & =\int_{\Gamma_{h}} v_{h} w_{h} \mathrm{~d} s_{h}, \quad \text { for all } v_{h}, w_{h} \in V_{h, m}, \\
\langle A V, W\rangle & =\int_{\Gamma_{h}} \nabla v_{h} \cdot \nabla w_{h} \mathrm{~d} s_{h}, \quad \text { for all } v_{h}, w_{h} \in V_{h, m}, \\
\left\langle A_{\Gamma} V, W\right\rangle & =\int_{\Gamma_{h}} \nabla_{\Gamma_{h}} v_{h} \cdot \nabla_{\Gamma_{h}} w_{h} \mathrm{~d} s_{h}, \quad \text { for all } v_{h}, w_{h} \in V_{h, m} .
\end{aligned}
$$


If $\left\{\left(\phi_{i}\right)_{\mid \Gamma_{h}}\right\}_{1 \leq i \leq N}$ are dependent, there exists $V \in \mathbb{R}^{N}, V \neq 0$, such that $v_{h}=$ $\sum_{i=1}^{N} V_{i} \phi_{i}=0$ on $\Gamma_{h}$. This implies $\langle M V, V\rangle=0$ and thus $M$ is singular. Furthermore, $v_{h \mid \Gamma_{h}}=0$ implies $\left(\nabla_{\Gamma_{h}} v_{h}\right)_{\mid \Gamma_{h}}=0$ and thus $\left\langle A_{\Gamma} V, V\right\rangle=0$, hence $A_{\Gamma}$ is singular. This indicates that the conditioning properties of the mass matrix $M$ and the stiffness matrix $A_{\Gamma}$ are different from that of standard finite element discretizations of elliptic problems. In [23] this conditioning issue is studied. We outline a few important results. In numerical experiments it is observed that for the Laplace-Beltrami equation discretized with linear trace finite elements on an approximate surface $\Gamma_{h}$ that is obtained as the zero level of a piecewise linear level set function the mass matrix $M$ has one zero eigenvalue (within machine accuracy) and the stiffness matrix $A_{\Gamma}$ has two zero eigenvalues. The effective condition number is defined as the quotient of the largest and the smallest nonzero eigenvalue. Typically both the diagonally scaled mass matrix $D_{M}^{-1} M$ and the diagonally scaled stiffness matrix $D_{A_{\Gamma}}^{-1} A_{\Gamma}$ have effective condition numbers that behave like $h^{-2}$. In [23] a rather technical analysis is presented that gives a theoretical explanation of these conditioning properties. The analysis is only for the two-dimensional case (i.e., $\Gamma$ is a curve) and uses technical assumptions related to how $\Gamma_{h}$ intersects the local triangulation $\mathcal{T}_{h}^{\Gamma}$. An example of such an assumption is that the relative size of the set of vertices in $\mathcal{T}_{h}^{\Gamma}$ having a certain maximal distance to $\Gamma_{h}$ gets smaller if this distance gets smaller (for precise statements we refer to [23]). In the recent paper [5] a stabilization technique for (2.6) is introduced, which improves the conditioning properties of $A_{\Gamma}$.

The discretization (2.5) is more stable than (2.6) in the sense that $\left\|\nabla v_{h}\right\|_{L^{2}\left(\Gamma_{h}\right)} \geq$ $\left\|\nabla_{\Gamma_{h}} v_{h}\right\|_{L^{2}\left(\Gamma_{h}\right)}$ holds. Related to this, note that $v_{h \mid \Gamma_{h}}=0$ does not necessarily imply that $\left(\nabla v_{h}\right)_{\mid \Gamma_{h}}=0$ holds. Based on this, one might expect a better conditioning of the matrix $A$ compared to $A_{\Gamma}$. This is indeed observed in numerical experiments, cf. section 7 . In this section we derive conditioning properties of the stiffness matrix $A$ for the case $m=1$, i.e., linear finite elements. Using an elementary analysis it is shown that a suitably scaled $A$ has a condition number that behaves like $h^{-2}$ (on the space orthogonal to the constant), independent of how $\Gamma_{h}$ intersects $\mathcal{T}_{h}^{\Gamma}$. Such a robustness property w.r.t. the geometry does not hold for the scaled stiffness matrix $A_{\Gamma}$. As a simple corollary we obtain a conditioning result for a shifted mass matrix, cf. Theorem 6.3.

We consider $m=1$ and use the notation $V_{h}:=V_{h, 1}$. In Remark 4 we comment on $m \geq 2$. For a node $x_{i}$, let $\mathcal{T}\left(x_{i}\right)$ be the set of all tetrahedra $T \in \mathcal{T}_{h}^{\Gamma}$ that contain $x_{i}$. Define

$$
\delta_{T}:=\frac{\left|\Gamma_{T}\right|}{|T|} h, \quad T \in \mathcal{T}_{h}^{\Gamma}, \quad d_{i}:=\sum_{T \in \mathcal{T}\left(x_{i}\right)} \delta_{T}, \quad 1 \leq i \leq N
$$

(Recall: $\left.\Gamma_{T}=\Gamma_{h} \cap T\right)$. We introduce a weighted $L^{2}\left(\omega_{h}\right)$-norm:

$$
\|v\|_{\delta, \omega_{h}}^{2}=\sum_{T \in \mathcal{T}_{h}^{\Gamma}} \delta_{T} \int_{T} v^{2} d x,
$$

and a related scaled vector norm:

$$
\|V\|_{D}^{2}:=\langle D V, V\rangle, \quad D:=\operatorname{diag}\left(d_{i}\right) .
$$

From the fact that $\nabla \phi_{i} \cdot \nabla \phi_{i}$ is constant on each $T \in \mathcal{T}\left(x_{i}\right)$ with value $\sim h^{-2}$ it follows that $D$ is uniformly spectrally equivalent to $\operatorname{diag}(A)$. Hence, the scaling with 
$D$ that is used below, can be replaced by a scaling with $\operatorname{diag}(A)$. The constants used in the lemma and theorems below are independent of $h$ and of how $\Gamma_{h}$ intersects the local triangulation $\mathcal{T}_{h}^{\Gamma}$.

LEMma 6.1. There are constants $c_{1}>0$ and $c_{2}$ such that

$$
c_{1} h^{3}\|V\|_{D}^{2} \leq\left\|v_{h}\right\|_{\delta, \omega_{h}}^{2} \leq c_{2} h^{3}\|V\|_{D}^{2} \quad \text { for all } v_{h} \in V_{h} .
$$

Proof. We use the compact notation $\sim$ to represent inequalities in both directions with constants independent of $h$ and of how $\Gamma_{h}$ intersects $\Omega_{h}$. The set of vertices of $T$ is denoted by $\mathcal{V}(T)$. For $v_{h} \in V_{h}$ we have $V_{i}=v_{h}\left(x_{i}\right)$ and

$$
\int_{T} v_{h}^{2} d x \sim|T| \sum_{x_{i} \in \mathcal{V}(T)} v_{h}\left(x_{i}\right)^{2} \sim h^{3} \sum_{x_{i} \in \mathcal{V}(T)} V_{i}^{2} .
$$

This implies

$$
\begin{aligned}
\left\|v_{h}\right\|_{\delta, \omega_{h}}^{2} & =\sum_{T \in \mathcal{T}_{h}^{\Gamma}} \delta_{T} \int_{T} v_{h}^{2} d x \sim h^{3} \sum_{T \in \mathcal{T}_{h}^{\Gamma}} \delta_{T} \sum_{x_{i} \in \mathcal{V}(T)} V_{i}^{2} \\
& =h^{3} \sum_{i=1}^{N}\left(\sum_{T \in \mathcal{T}\left(x_{i}\right)} \delta_{T}\right) V_{i}^{2}=h^{3} \sum_{i=1}^{N} d_{i} V_{i}^{2}=h^{3}\|V\|_{D}^{2},
\end{aligned}
$$

and thus the result holds. $\square$

THEOREM 6.2. There are constants $c_{1}>0$ and $c_{2}$ such that

$$
c_{1} h^{2}\|V\|_{D}^{2} \leq\langle A V, V\rangle \leq c_{2}\|V\|_{D}^{2} \quad \text { for all } V \in \mathbb{R}^{N} \text { with } \int_{\Gamma_{h}} v_{h} d s=0 .
$$

Proof. We first consider the upper bound. Note that, using an inverse inequality on $T$ we get:

$$
\begin{aligned}
\langle A V, V\rangle & =\int_{\Gamma_{h}} \nabla v_{h} \cdot \nabla v_{h} d s \leq \sum_{T \in \mathcal{T}_{h}^{\Gamma}}\left|\Gamma_{T}\right|\left\|\nabla v_{h}\right\|_{L^{\infty}(T)}^{2} \leq c \sum_{T \in \mathcal{T}_{h}^{\Gamma}} h^{-2} \frac{\left|\Gamma_{T}\right|}{|T|}\left\|v_{h}\right\|_{L^{2}(T)}^{2} \\
& =c h^{-3} \sum_{T \in \mathcal{T}_{h}^{\Gamma}} \delta_{T} \int_{T} v_{h}^{2} d x=c h^{-3}\left\|v_{h}\right\|_{\delta, \omega_{h}}^{2} \leq c\|V\|_{D}^{2},
\end{aligned}
$$

where in the last inequality we used Lemma 6.1.

We now consider the lower bound. Using Lemma 6.1 we get

$$
h^{2}\|V\|_{D}^{2} \leq c h^{-1}\left\|v_{h}\right\|_{\delta, \omega_{h}}^{2}=c h^{-1} \sum_{T \in \mathcal{T}_{h}^{\Gamma}} \delta_{T} \int_{T} v_{h}^{2} d x .
$$

Take a $T \in \mathcal{T}_{h}^{\Gamma}$. Let $\xi \in T, \eta \in \Gamma_{T}$ be such that $\left|v_{h}(x)\right| \leq\left|v_{h}(\xi)\right|$ for all $x \in T$ and $\left|v_{h}(\eta)\right| \leq\left|v_{h}(x)\right|$ for all $x \in \Gamma_{T}$. From $v_{h}(\xi)=v_{h}(\eta)+(\xi-\eta) \cdot \nabla v_{h \mid T}$ it follows that $\left|v_{h}(\xi)\right|^{2} \leq c\left(\left|v_{h}(\eta)\right|^{2}+h^{2}\left\|\nabla v_{h \mid T}\right\|^{2}\right)$. Using this we obtain

$$
\begin{aligned}
h^{-1} \delta_{T} \int_{T} v_{h}^{2} d x & \leq\left|\Gamma_{T} \| v_{h}(\xi)\right|^{2} \leq c\left(\left|\Gamma_{T}\left\|\left.v_{h}(\eta)\right|^{2}+\left|\Gamma_{T}\right| h^{2}\right\| \nabla v_{h \mid T} \|^{2}\right)\right. \\
& \leq c\left(\int_{\Gamma_{T}} v_{h}^{2} d s+h^{2} \int_{\Gamma_{T}}\left\|\nabla v_{h}\right\|^{2} d s .\right)
\end{aligned}
$$


Summing over $T \in \mathcal{T}_{h}^{\Gamma}$ and using the Poincare inequality (5.3) (which holds for $v_{h}$ with $\left.\int_{\Gamma_{h}} v_{h} \mathrm{~d} s_{h}=0\right)$, we get

$$
\begin{aligned}
& h^{-1} \sum_{T \in \mathcal{T}_{h}^{\Gamma}} \delta_{T} \int_{T} v_{h}^{2} d x \leq c\left(\int_{\Gamma_{h}} v_{h}^{2} d s+h^{2} \int_{\Gamma_{h}}\left\|\nabla v_{h}\right\|^{2} d s\right) \\
& \leq c\left(\int_{\Gamma_{h}}\left\|\nabla_{\Gamma_{h}} v_{h}\right\|^{2} d s+h^{2} \int_{\Gamma_{h}}\left\|\nabla v_{h}\right\|^{2} d s\right) \leq c \int_{\Gamma_{h}}\left\|\nabla v_{h}\right\|^{2} d s=c\langle A V, V\rangle .
\end{aligned}
$$

Combining this with the result in (6.4) completes the proof. $\square$

As an immediate conseqence of this theorem we obtain for the spectral condition number in the space orthogonal to the one dimensional kernel (corresponding to the constant fuction), denoted by $\kappa_{*}(\cdot)$ :

$$
\kappa_{*}\left(D^{-1} A\right) \leq c h^{-2} .
$$

We derive a result for a shifted mass matrix.

THEOREM 6.3. There are constants $c_{1}, c_{2}>0$ such that for all $\alpha \geq 0$ :

$$
c_{1}\left(\min \left\{2 h^{2}, \alpha\right\}+\alpha h^{2}\right)\|V\|_{D}^{2} \leq\langle M V, V\rangle+\alpha\langle A V, V\rangle \leq c_{2}\left(h^{2}+\alpha\right)\|V\|_{D}^{2},
$$

for all $V \in \mathbb{R}^{N}$ with $\int_{\Gamma_{h}} v_{h} d s=0$.

Proof. First we consider the upper bound. Note that

$$
\begin{aligned}
\langle M V, V\rangle & =\int_{\Gamma_{h}} v_{h}^{2} d s \leq \sum_{T \in \mathcal{T}_{h}^{\Gamma}}\left|\Gamma_{T}\right|\left\|v_{h}\right\|_{L^{\infty}(T)}^{2} \leq c h^{-1} \sum_{T \in \mathcal{T}_{h}^{\Gamma}} \delta_{T} \int_{T} v_{h}^{2} d s \\
& =c h^{-1}\left\|v_{h}\right\|_{\delta, \omega_{h}}^{2} \leq c h^{2}\|V\|_{D}^{2} .
\end{aligned}
$$

In combination with Theorem 6.2 this yields the upper bound. For the lower bound we use the result in (6.5) and in Lemma 6.1 and thus get:

$$
\langle M V, V\rangle+h^{2}\langle A V, V\rangle=\int_{\Gamma_{h}} v_{h}^{2} d s+h^{2} \int_{\Gamma_{h}}\left\|\nabla v_{h}\right\|^{2} d s \geq c h^{-1}\left\|v_{h}\right\|_{\delta, \omega_{h}}^{2} \geq c_{0} h^{2}\langle D V, V\rangle,
$$

with a constant $c_{0}>0$. Using spectral inequalities for symmetric positive matrices we have: $M+h^{2} A \geq c_{0} h^{2} D$. From this and the lower bound in Theorem 6.2 we get

$$
\begin{aligned}
M+\alpha A & =M+\left(\frac{\alpha}{2 h^{2}}\right) h^{2} A+\frac{1}{2} \alpha A \geq \min \left\{1, \frac{\alpha}{2 h^{2}}\right\}\left(M+h^{2} A\right)+\frac{1}{2} \alpha A \\
& \geq c_{0} \min \left\{1, \frac{\alpha}{2 h^{2}}\right\} h^{2} D+c_{1} \alpha h^{2} D \geq c\left(\min \left\{2 h^{2}, \alpha\right\}+\alpha h^{2}\right) D,
\end{aligned}
$$

which proves the lower bound.

Thus we have the following bound for the spectral condition number:

$$
\kappa_{*}\left(D^{-1}(M+\alpha A)\right) \leq c \frac{h^{2}+\alpha}{\min \left\{2 h^{2}, \alpha\right\}+\alpha h^{2}} .
$$

For $\alpha \rightarrow \infty$ we get the same bound as in (6.6). For $\alpha \downarrow 0$ the bound tends to infinity. This can not be avoided, since the mass matrix $M$ can be singular (also in the space orthogonal to the constant), as explained above. More interesting is the case $\alpha \geq c h^{2}$ with $c>0$. Then the bound takes the form $c h^{-2} \frac{\alpha}{1+\alpha}$. Hence, if $\alpha \sim h^{2}$ we get a 
uniform (i.e. independent of $h$ ) condition number bound and if $\alpha \sim h$ we get a bound of the form $c h^{-1}$. The case $\alpha \sim h^{2}$ is typical if a time dependent surface diffusion problem is considered in which for the time discretization an implicit Euler method is used with $\Delta t \sim h^{2}$. If for such a time dependent problem one uses Crank-Nicolson with $\Delta t \sim h$, this results in a linear system with $\alpha \sim h$.

REMARK 4 . We comment on the case of higher order finite elements, i.e. $m \geq 2$. Inspection of the proofs, shows that the estimates in Lemma 6.1 and the upper bound in Theorem 6.2 also hold if $m \geq 2$ is considered. The lower bound in Theorem 6.2, however, does not hold in general. This becomes clear from the following example. Consider a two-dimensional setting with a domain $\omega=[0,1] \times[-1,1]$ which is subdivided into a few triangles with vertices only on $y=-1$ or $y=1$. We take $\Gamma_{h}=[0,1] \times\{0\}$. We choose the $P_{2}$ finite element function $v(x, y)=\alpha y^{2}+\left(x-\frac{1}{2}\right)$ with $\alpha \gg 1$. For this function we have $\|v\|_{L^{2}(\omega)} \sim \alpha, \int_{\Gamma_{h}} v \mathrm{~d} s=0, \nabla v=(1,2 \alpha y)^{T}$ and thus $\|\nabla v\|_{L^{2}\left(\Gamma_{h}\right)}=1$. The lower bound in Theorem 6.2 scales with $\|v\|_{L^{2}(\omega)}^{2} \sim \alpha^{2}$, whereas $\langle A V, V\rangle=\|\nabla v\|_{L^{2}\left(\Gamma_{h}\right)}^{2}=1$ holds. Hence, we conclude that the first inequality in Theorem ?? can not hold for this simple example. The key point in this example is that we can not control the values of $v_{h}$ on $\omega$ by its values and gradient on $\Gamma_{h}$.

7. Numerical experiments. In this section we present results of a numerical experiment. As a test problem we consider the Laplace-Beltrami equation on the unit sphere:

$$
-\Delta_{\Gamma} u=f \quad \text { on } \Gamma
$$

with $\Gamma=\left\{x \in \mathbb{R}^{3} \mid\|x\|_{2}=1\right\}$ and $\Omega=(-2,2)^{3}$.

The source term $f$ is taken such that the solution is given by

$$
u(x)=\frac{1}{\|x\|^{3}}\left(3 x_{1}^{2} x_{2}-x_{2}^{3}\right), \quad x=\left(x_{1}, x_{2}, x_{3}\right) \in \Omega .
$$

Using the representation of $u$ in spherical coordinates one can verify that $u$ is an eigenfunction of $-\Delta_{\Gamma}$ :

$$
u(r, \phi, \theta)=\sin (3 \phi) \sin ^{3} \theta, \quad-\Delta_{\Gamma} u=12 u=: f(r, \phi, \theta) .
$$

The right-hand side $f$ satisfies the compatibility condition $\int_{\Gamma} f \mathrm{~d} s=0$, likewise does $u$. Note that $u$ and $f$ are constant along normals at $\Gamma$.

A family $\left\{\mathcal{T}_{l}\right\}_{l \geq 0}$ of tetrahedral triangulations of $\Omega$ is constructed as follows. We triangulate $\Omega$ by starting with a uniform subdivision into 48 tetrahedra with mesh size $h_{0}=\sqrt{3}$. Then we apply an adaptive red-green refinement-algorithm (implemented in the software package DROPS [11]) in which in each refinement step the tetrahedra that contain $\Gamma$ are refined such that on level $l=1,2, \ldots$ we have

$$
h_{T} \leq \sqrt{3} 2^{-l}=: h_{l} \quad \text { for all } T \in \mathcal{T}_{l} \text { with } T \cap \Gamma \neq \emptyset .
$$

The family $\left\{\mathcal{T}_{l}\right\}_{l \geq 0}$ is consistent and shape-regular. The interface $\Gamma$ is the zero-level of $\varphi(x):=\|x\|^{2}-1$. Let $\varphi_{h}:=I_{h}(\varphi)$ where $I_{h}$ is the standard nodal interpolation operator on $\mathcal{T}_{l}$. The discrete interface is given by $\Gamma_{h_{l}}:=\left\{x \in \Omega \mid I\left(\phi_{h}\right)(x)=0\right\}$. This discrete interface triangulation is very shape-irregular. For the extension $f_{h}$ of $f$ we take the constant extension of $f$ along the normals at $\Gamma$, i.e. we take $f_{h}(r, \phi, \theta)=$ $f(1, \phi, \theta)+c_{h}$, with $f(r, \phi, \theta)$ as in (7.1) and $c_{h}$ such that $\int_{\Gamma_{h}} f_{h} \mathrm{~d} s_{h}=0$. For the 
computation of the integrals $\int_{T} f_{h} \phi_{h} \mathrm{~d} s_{h}$ we use a quadrature-rule that is exact up to order five.

We consider the discrete problems (2.5) and (2.6) with solutions $u_{h}$ and $u_{h}^{\Gamma}$, respectively.

The discretization errors in the $L^{2}\left(\Gamma_{h}\right)$-norm are given in table 7.1. The extension $u^{e}$ of $u$ is given by $u^{e}(r, \phi, \theta):=u(1, \phi, \theta)=u(r, \phi, \theta)$ (since $u$ is constant along $n_{\Gamma}$ ).

\begin{tabular}{|rl|r|r||r|r|}
\hline level & $l$ & $\left\|u^{e}-u_{h}\right\|_{L^{2}\left(\Gamma_{h}\right)}$ & factor & $\left\|u^{e}-u_{h}^{\Gamma}\right\|_{L^{2}\left(\Gamma_{h}\right)}$ & factor \\
\hline & 1 & 0.6276 & - & 0.4418 & - \\
& 0.1983 & 3.16 & 0.1149 & 3.85 \\
& 0.05299 & 3.74 & 0.02965 & 3.87 \\
& 0.01348 & 3.93 & 0.007298 & 4.06 \\
& 0.003387 & 3.98 & 0.001865 & 3.91 \\
5 & 0.0008476 & 4.00 & 0.0004629 & 4.03 \\
& 0.0002120 & 4.00 & 0.0001158 & 4.00 \\
\hline & \multicolumn{4}{|c}{ TABLE 7.1} &
\end{tabular}

Discretization errors and error reduction.

These results clearly show the $h^{2}$ behaviour as predicted by the analysis. We also observe that the discretization error for $u_{h}^{\Gamma}$ is about a factor two smaller than for $u_{h}$.

We now consider the conditioning of the stiffness matrices and shifted mass matrix. The matrices $M, A, A_{\Gamma}$ and $D$ are as defined in section 6. Define $D_{A_{\Gamma}}:=\operatorname{diag}\left(A_{\Gamma}\right)$ and the scaled matrices

$$
\tilde{A}:=D^{-\frac{1}{2}} A D^{-\frac{1}{2}}, \quad \tilde{A}_{\Gamma}:=D_{A_{\Gamma}}^{-\frac{1}{2}} A_{\Gamma} D_{A_{\Gamma}}^{-\frac{1}{2}}, \quad \tilde{M}_{\alpha}:=D^{-\frac{1}{2}}(M+\alpha A) D^{-\frac{1}{2}} .
$$

The discrete problems are solved using a standard CG method with symmetric SOR preconditioner applied to the discrete problems with stiffness matrices $A$ and $A_{\Gamma}$. We use a relative tolerance of $10^{-6}$. In the tables below $m$ gives the number of unknows (dimension of the matrices). In the tables 7.2 and 7.3 "\# iter" gives the number of preconditioned CG iterations needed to solve the system (with accuracy $10^{-6}$ ). Furthermore, for different refinement levels we computed the largest and smallest eigenvalues of scaled the matrices.

\begin{tabular}{|c|c|c|l|l|l|c|c|c|}
\hline level $l$ & $m$ & factor & $\lambda_{1}$ & $\lambda_{2}$ & $\lambda_{m}$ & $\lambda_{m} / \lambda_{2}$ & factor & $\#$ iter \\
\hline 1 & 100 & - & 0 & 0.021 & 0.51 & 24.0 & - & 11 \\
2 & 448 & 4.48 & 0 & 0.0053 & 0.52 & 98.9 & 4.1 & 18 \\
3 & 1864 & 4.16 & 0 & 0.0013 & 0.54 & 412 & 4.2 & 33 \\
4 & 7552 & 4.05 & 0 & 0.00033 & 0.54 & 1667 & 4.0 & 59 \\
\hline \multicolumn{7}{c}{ TABLE 7.2 } \\
Conditioning of scaled stiffness matrix $\tilde{A}$
\end{tabular}

We observe that, as predicted by the theory, the effective condition number for $\tilde{A}$ behaves like $\sim h^{-2}$ and that this condition number is smaller the one of $\tilde{A}_{\Gamma}$. The better conditioning of $\tilde{A}$ is also reflected in the results for \# iter. Also note that $\tilde{A}$ has only one zero eigenvalue (corresponding to the constant function), whereas $\tilde{A}_{\Gamma}$ 


\begin{tabular}{|c|c|c|c|l|l|l|c|c|c|}
\hline level $l$ & $m$ & factor & $\lambda_{1}$ & $\lambda_{2}$ & $\lambda_{3}$ & $\lambda_{m}$ & $\lambda_{m} / \lambda_{3}$ & factor & \# iter \\
\hline 1 & 100 & - & 0 & 0 & 0.055 & 2.16 & 39.2 & - & 13 \\
2 & 448 & 4.48 & 0 & 0 & 0.014 & 2.19 & 154 & 3.9 & 25 \\
3 & 1864 & 4.16 & 0 & 0 & 0.0033 & 2.34 & 710 & 4.6 & 49 \\
4 & 7552 & 4.05 & 0 & 0 & 0.00077 & 2.43 & 3150 & 4.4 & 98 \\
\hline
\end{tabular}

Conditioning of scaled stiffness matrix $\tilde{A}_{\Gamma}$

has two zero eigenvalues. In Table 7.4 we present results for the spectral condition number $\kappa_{*}\left(\tilde{M}_{\alpha}\right)$ for the cases $\alpha=h_{l}^{2}$ and $\alpha=h_{l}$. The results are in very good agreement with the bound derived in (6.7).

\begin{tabular}{|c|c|c|c|}
\hline level $l$ & $m$ & $\kappa_{*}\left(\tilde{M}_{\alpha}\right), \alpha=h_{l}^{2}$ & $\kappa_{*}\left(\tilde{M}_{\alpha}\right), \alpha=h_{l}$ \\
\hline 1 & 100 & 12.7 & 25.0 \\
2 & 448 & 13.1 & 50.7 \\
3 & 1864 & 13.5 & 104 \\
4 & 7552 & 13.6 & 209 \\
\hline
\end{tabular}

Conditioning of scaled shifted mass matrix $\tilde{M}_{\alpha}$

\section{REFERENCES}

[1] D. Adalsteinsson and J. A. Sethian, Transport and diffusion of material quantities on propagating interfaces via level set methods, J. Comput. Phys., 185 (2003), pp. 271-288.

[2] B. Alberta, A. Johnson, J. Lewis, M. Raff, K. Roberts, and P. Walter, Molecular Biology of the Cell, Garland Science, New York, fouth ed., 2002.

[3] D. Braess, Finite Elements: Theory, Fast Solvers, and Applications in Solid Mechanics, $3 d$ edition, Cambridge University Press, 2007.

[4] L. Brenner, S.and Scott, The Mathematical Theory of Finite Element Methods, Springer, New York, second ed., 2002.

[5] E. Burman, P. Hansbo, and M. Larson, A stable cut finite element method for partial differential equations on surfaces: The Laplace-Beltrami operator, preprint, arXiv:1312.1097, 2013.

[6] A. Chernyshenko and M. Olshanskit, Non-degenerate Eulerian finite element method for solving PDEs on surfaces, Rus. J. Num. Anal. Math. Model., 28 (2013), pp. 101-124.

[7] K. Deckelnick, C. Elliott, and T. Ranner, Unfitted finite element methods using bulk meshes for surface partial differential equations, preprint, arXiv:1312.2905, 2013.

[8] A. Demlow, Higher-order finite element methods and pointwise error estimates for elliptic problems on surfaces, SIAM J. Numer. Anal., 47 (2009), pp. 805-827.

[9] A. Demlow and G. Dziuk, An adaptive finite element method for the Laplace-Beltrami operator on implicitly defined surfaces, SIAM J. Numer. Anal., 45 (2007), pp. 421-442.

[10] A. Demlow and M. OlshanskiI, An adaptive surface finite element method based on volume meshes, SIAM J. Numer. Anal., 50 (2012), pp. 1624-1647.

[11] DROPS package. http://www.igpm.rwth-aachen.de/DROPS/.

[12] G. Dziuk, Finite elements for the beltrami operator on arbitrary surfaces, in Partial differential equations and calculus of variations, S. Hildebrandt and R. Leis, eds., vol. 1357 of Lecture Notes in Mathematics, Springer, 1988, pp. 142-155.

[13] G. Dziuk and C. Elliott, Finite elements on evolving surfaces, IMA J. Numer. Anal., 27 (2007), pp. 262-292.

[14] - An Eulerian approach to transport and diffusion on evolving implicit surfaces, Comput Visual Sci, 13 (2010), pp. 17-28. 
[15] C. M. Elliott, B. Stinner, V. Styles, and R. Welford, Numerical computation of advection and diffusion on evolving diffuse interfaces, IMA J. Numer. Anal., 31 (2011), pp. $786-812$.

[16] J. B. Greer, An improvement of a recent Eulerian method for solving PDEs on general geometries, J. Sci. Comput., 29 (2008), pp. 321-352.

[17] S. Gross And A. Reusken, Numerical Methods for Two-phase Incompressible Flows, Springer, Berlin, 2011.

[18] A. Hansbo And P. HANSBO, An unfitted finite element method, based on nitsche's method, for elliptic interface problems, Comput. Methods Appl. Mech. Engrg., 191 (2002), pp. 55375552 .

[19] — A finite element method for the simulation of strong and weak discontinuities in solid mechanics, Comput. Methods Appl. Mech. Engrg., 193 (2004), pp. 3523-3540.

[20] M. Olshanski And A. Reusken, Error analysis of a space-time finite element method for solving PDEs on evolving surfaces, IGPM Preprint No 376, Department of Mathematics, RWTH Aachen University. Revised version submitted to SIAM J. Numer. Anal., (2013).

[21] M. Olshanskit, A. Reusken, And X. Xu, An Eulerian space-time finite element method for diffusion problems on evolving surfaces, NA\&SC Preprint No 5, Department of Mathematics, University of Houston. Revised version submitted to SIAM J. Numer. Anal., (2013).

$[22][-$ A stabilized finite element method for advection-diffusion equations on surfaces, IMA J. of Numer. Anal., doi:10.1093/imanum/drt016, (2013).

[23] M. A. Olshanskit and A. Reusken, A finite element method for surface PDEs: Matrix properties, Numer. Math., 114 (2010), pp. 491-520.

[24] M. A. Olshanskit, A. Reusken, and J. Grande, A finite element method for elliptic equations on surfaces, SIAM J. Numer. Anal., 47 (2009), pp. 3339-3358.

[25] T. Ranner, Computational surface partial differential equations, PhD thesis, University of Warwick, 2013.

[26] J. A. Sethian, Theory, algorithms, and applications of level set methods for propagating interfaces, Acta Numerica, 5 (1996), pp. 309-395.

[27] J.-J. XU AND H.-K. ZHAO, An Eulerian formulation for solving partial differential equations along a moving interface, Journal of Scientific Computing, 19 (2003), pp. 573-594. 